\title{
New characterization and parametrization of LCD Codes
}

\author{
Claude Carlet $^{1}$ Sihem Mesnager ${ }^{2}$ Chunming Tang ${ }^{3}$ Yanfeng Qi ${ }^{4}$
}

\begin{abstract}
Linear complementary dual (LCD) cyclic codes were referred historically to as reversible cyclic codes, which had applications in data storage. Due to a newly discovered application in cryptography, there has been renewed interest in LCD codes. In particular, it has been shown that binary LCD codes play an important role in implementations against side-channel attacks and fault injection attacks. In this paper, we first present a new characterization of binary LCD codes in terms of their symplectic basis. Using such a characterization,we solve a conjecture proposed by Galvez et al. on the minimum distance of binary LCD codes. Next, we consider the action of the orthogonal group on the set of all LCD codes, determine all possible orbits of this action, derive simple closed formulas of the size of the orbits, and present some asymptotic results of the size of the corresponding orbits. Our results show that almost all binary LCD codes are odd-like codes with odd-like duals, and about half of $q$-ary LCD codes have orthonormal basis, where $q$ is a power of an odd prime.
\end{abstract}

\section{Index Terms}

LCD codes, Odd-like LCD codes, Even-like LCD codes, Group action, Orthogonal group, Symplectic group.

\section{INTRODUCTION}

Let $q$ be a power of a prime. $\mathbb{F}_{q}$ and $\mathbb{F}_{q}^{n}$ denote the finite field with $q$ elements and $n$-dimensional vector space over $\mathbb{F}_{q}$ respectively. Let $\mathrm{wt}(\mathbf{x})$ denote the weight of $\mathbf{x} \in \mathbb{F}_{q}^{n}$, i.e., the number of nonzero elements in $\mathbf{x}=\left(x_{1}, x_{2}, \ldots, x_{n}\right)$. For any $\mathbf{x}=\left(x_{1}, x_{2}, \ldots, x_{n}\right)$ and $\mathbf{y}=\left(y_{1}, y_{2}, \ldots, y_{n}\right)$ in $\mathbb{F}_{q}^{n}$, the Euclidean inner product of $\mathbf{x}$ and $\mathbf{y}$ is defined by

$$
\mathbf{x} \cdot \mathbf{y}=\sum_{i=1}^{n} x_{i} y_{i}
$$

An $[n, k, d]$ linear code $\mathcal{C}$ over $\mathbb{F}_{q}$ is a $k$-dimensional subspace of $\mathbb{F}_{q}^{n}$ with minimum (Hamming) distance $d$. The $d u a l$ of $\mathcal{C}$ is defined by

$$
\mathcal{C}^{\perp}=\left\{\mathbf{w} \in \mathbb{F}_{q}^{n}: \mathbf{w} \cdot \mathbf{c}=0 \text { for all } \mathbf{c} \in \mathcal{C}\right\} .
$$

If $\mathcal{C} \cap \mathcal{C}^{\perp}=\{\mathbf{0}\}$, then $\mathcal{C}$ is called a linear complementary dual code or an $L C D$ code.

Carlet and Guilley applied LCD codes in side-channel attacks (SCA) and fault non-invasive attacks [1], [2]. A lot of works has been devoted to construct LCD codes. In [5], Ding et al. constructed several families of Euclidean LCD cyclic codes over finite fields and analyzed their parameters. In [9] $\mathrm{Li}$ et al. studied two special families of LCD cyclic codes, which are both $\mathrm{BCH}$ codes. Mesnager et al. [10] presented a construction of algebraic geometry Euclidean LCD codes. In [3], Carlet et al. completely determined all $q$-ary $(q>3)$ Euclidean LCD codes and all $q^{2}$-ary $(q>2)$ Hermitian LCD codes for all possible parameters. In the latest paper, Carlet et al. [4], introduced the concept of linear codes with $\sigma$ complementary dual ( $\sigma$-LCD), which includes known Euclidean LCD codes, Hermitian LCD codes, and Galois LCD codes. Their results extend those on the classical LCD codes and show that $\sigma$-LCD codes allow the construction of LCP of codes more easily and with more flexibility.

However, little is known on the general structure of LCD codes. The goal of this paper is to characterize LCD codes and to study the structure of the set of LCD codes. We first present a new characterization of LCD codes in terms of their symplectic basis. Such a characterization, allows us to solve a conjecture proposed by Galvez et al. [7] on the minimum distance of binary LCD codes. Afterwards, we consider the action of the orthogonal group on the set of all LCD codes, determine all possible orbits of this action, give simple closed formulas of the size of the orbits, and present some asymptotic results of the size of the corresponding orbits. Our results show that almost all binary LCD codes are odd-like codes with odd-like duals, and about half of $q$-ary LCD codes have orthogonal basis, where $q$ is a power of an odd prime.

This work was supported by SECODE project and the National Natural Science Foundation of China (Grant No. 11401480, 11531002, 11701129). C. Tang also acknowledges support from 14E013, CXTD2014-4 and the Meritocracy Research Funds of China West Normal University. Y. Qi also acknowledges support from Zhejiang provincial Natural Science Foundation of China (LQ17A010008 and LQ16A010005).

C. Carlet is with the Department of Mathematics, University of Paris VIII, 93526 Saint-Denis, France and also with University of Paris XIII, CNRS, LAGA UMR 7539, Sorbonne Paris Cité, 93430 Villetaneuse, France (email: claude.carlet@univ-paris8.fr).

S. Mesnager is with the Department of Mathematics, University of Paris VIII, 93526 Saint-Denis, France, also with University of Paris XIII, CNRS, LAGA UMR 7539, Sorbonne Paris Cité, 93430 Villetaneuse, France, and also with Telecom ParisTech 75013 Paris (email: smesnager@univ-paris8.fr).

C. Tang is with School of Mathematics and Information, China West Normal University, Nanchong, Sichuan, 637002, China. e-mail: tangchunmingmath@163.com

Y. Qi is with School of Science, Hangzhou Dianzi University, Hangzhou, Zhejiang, 310018, China. e-mail: qiyanfeng07@163.com 
The paper is organized as follows. In Section [I] we recall some basic results on LCD codes and binary symmetric matrices. In Section III], we firstly characterize binary LCD codes in terms of their basis. Based on these results we solve a conjecture proposed by Galvez et al. [7]. In Section IV] we consider the action of orthogonal group on the set of all binary LCD codes and study the orbits of this action. In Section $\nabla$, we use the same method to characterize $q$-ary LCD codes, where $q$ is a power of an odd prime.

\section{PReliminaries} [11].

For a matrix $G, G^{T}$ denotes the transposed matrix of $G$. The following characterization of LCD codes is due to Massey

Proposition 2.1: Let $\mathcal{C}$ be a linear code with a generator matrix $G$ and a parity-check matrix $H$. Then the three following properties are equivalent:

(i) $\mathcal{C}$ is $\mathrm{LCD}$;

(ii) the matrix $G G^{T}$ is invertible;

(iii) the matrix $H H^{T}$ is invertible.

A matrix $M$ is symmetric if $M^{T}=M$. A diagonal matrix is a matrix in which the entries outside the main diagonal are all zero. We shall write $\operatorname{diag}\left[x_{1}, \ldots, x_{k}\right]$ for a diagonal matrix whose main diagonal entries are $x_{1}, \ldots, x_{k}$. The following proposition gives the classification of symmetric matrices over $\mathbb{F}_{2}$ under the equivalence relation $M \sim Q M Q^{T}$ [15], [16], where $Q$ is nonsingular.

Proposition 2.2: Let $M$ be a symmetric $k \times k$ matrix of rank $t$ with entries in $\mathbb{F}_{2}$.

(i) If all diagonal entries of $M$ equal 0 , then $t$ is even and there is a nonsingular matrix $Q$ such that

$$
Q M Q^{T}=\operatorname{diag}[\underbrace{J_{2}, J_{2}, \ldots, J_{2}}_{t}, 0, \ldots, 0],
$$

where $J_{2}=\left[\begin{array}{ll}0 & 1 \\ 1 & 0\end{array}\right]$.

(ii) If at least one diagonal entry of $M$ is nonzero, then there is a nonsingular $k \times k$ matrix $Q$ such that

$$
Q M Q^{T}=\operatorname{diag}[\underbrace{1,1, \ldots, 1}_{t}, 0, \ldots, 0] .
$$

\section{NEW CHARACTERIZATION OF BINARY LCD CODES BY THEIR BASIS}

In this section, we will present a new characterization of binary LCD codes. Based on this characterization, we prove a conjecture on minimum distance of binary LCD codes proposed by Galvez et al. [7].

A vector $\mathbf{x}=\left(x_{1}, x_{2}, \ldots, x_{n}\right)$ in $\mathbb{F}_{2}^{n}$ is even-like if

$$
\sum_{i=1}^{n} x_{i}=0
$$

and is odd-like otherwise. A code is said to be even-like if it has only even-like codewords, and is said to be odd-like if it is not even-like.

Theorem 3.1: Let $\mathcal{C}$ be an odd-like binary code with parameters $[n, k]$. Then $\mathcal{C}$ is LCD if and only if there exists a basis $\mathbf{c}_{1}, \mathbf{c}_{2}, \ldots, \mathbf{c}_{k}$ of $\mathcal{C}$ such that for any $i, j \in\{1,2, \ldots, k\}, \mathbf{c}_{i} \cdot \mathbf{c}_{j}$ equals 1 if $i=j$ and equals 0 if $i \neq j$.

Proof If there exists a basis $\mathbf{c}_{1}, \mathbf{c}_{2}, \ldots, \mathbf{c}_{k}$ of $\mathcal{C}$ such that for any $i, j \in\{1,2, \ldots, k\}, \mathbf{c}_{i} \cdot \mathbf{c}_{j}$ equals 1 if $i=j$ and equals 0 if $i \neq j$. Let $G$ be the generator matrix of $\mathcal{C}$ that corresponds to the base $\mathbf{c}_{1}, \mathbf{c}_{2}, \ldots, \mathbf{c}_{k}$. Then, $G G^{T}=I_{k}$, where $I_{k}$ is the $k \times k$ identity matrix. From Proposition $2.1 \mathcal{C}$ is LCD.

Conversely, assume that $\mathcal{C}$ is LCD. Let $G^{\prime}$ be a generator matrix of $\mathcal{C}$. Then from Proposition 2.1, $G^{\prime} G^{\prime T}$ is an invertible symmetric matrix of size $k \times k$. Since $\mathcal{C}$ is odd-like, there is at least one nonzero diagonal entry on $G^{\prime} G^{\prime T}$. Then from Part (ii) of Proposition 2.2 there exists nonsingular $k \times k$ matrix $Q$ such that $Q G^{\prime} G^{\prime T} Q^{T}=\left(Q G^{\prime}\right)\left(Q G^{\prime}\right)^{T}=I_{k}$, where $I_{k}$ is the $k \times k$ identity matrix. Let $G=Q G^{\prime}$. Then, $G$ is also a generator matrix of $\mathcal{C}$. Let $\mathbf{c}_{i}$ be the $i$-th row of matrix $G$ for $i \in\{1,2, \ldots, k\}$. Hence $\mathbf{c}_{1}, \mathbf{c}_{2}, \ldots, \mathbf{c}_{k}$ is the desired basis, since $G G^{T}=I_{k}$. It completes the proof.

Remark 1: Theorem 3.1 shows that a binary odd-like code $\mathcal{C}$ is LCD if and only if $\mathcal{C}$ has an orthonormal basis.

Theorem 3.2: Let $\mathcal{C}$ be an even-like binary code with parameters $[n, k]$. Then $\mathcal{C}$ is LCD if and only if $k$ is even and there exists a basis $\mathbf{c}_{1}, \mathbf{c}_{1}^{\prime}, \mathbf{c}_{2}, \mathbf{c}_{2}^{\prime}, \ldots, \mathbf{c}_{\frac{k}{2}}, \mathbf{c}_{\frac{k}{2}}^{\prime}$ of $\mathcal{C}$ such that for any $i, j \in\left\{1,2, \ldots, \frac{k}{2}\right\}$, the following conditions hold

(i) $\mathbf{c}_{i} \cdot \mathbf{c}_{j}=\mathbf{c}_{i}^{\prime} \cdot \mathbf{c}_{j}^{\prime}=0$

(ii) $\mathbf{c}_{i} \cdot \mathbf{c}_{j}^{\prime}=0$ if $i \neq j$;

(iii) $\mathbf{c}_{i} \cdot \mathbf{c}_{i}^{\prime}=1$. 
Proof If $k$ is even and there exists a basis $\mathbf{c}_{1}, \mathbf{c}_{1}^{\prime}, \mathbf{c}_{2}, \mathbf{c}_{2}^{\prime}, \ldots, \mathbf{c}_{\frac{k}{2}}, \mathbf{c}_{\frac{k}{2}}^{\prime}$ of $\mathcal{C}$ such that for any $i, j \in\left\{1,2, \ldots, \frac{k}{2}\right\}$, the following conditions hold

(i) $\mathbf{c}_{i} \cdot \mathbf{c}_{j}=\mathbf{c}_{i}^{\prime} \cdot \mathbf{c}_{j}^{\prime}=0$;

(ii) $\mathbf{c}_{i} \cdot \mathbf{c}_{j}^{\prime}=0$ if $i \neq j$;

(iii) $\mathbf{c}_{i} \cdot \mathbf{c}_{i}^{\prime}=1$.

Let $G$ be the generator matrix of $\mathcal{C}$ that corresponds to the base $\mathbf{c}_{1}, \mathbf{c}_{1}^{\prime}, \mathbf{c}_{2}, \mathbf{c}_{2}^{\prime}, \ldots, \mathbf{c}_{\frac{k}{2}}, \mathbf{c}_{\frac{k}{2}}^{\prime}$. Then, $G G^{T}=\operatorname{diag}[\underbrace{J_{2}, J_{2}, \ldots, J_{2}}_{\frac{k}{2}}]$, where $J_{2}=\left[\begin{array}{ll}0 & 1 \\ 1 & 0\end{array}\right]$. Hence, $G G^{T}$ is invertible. From Proposition 2.1, we have that $\mathcal{C}$ is LCD.

Conversely, assume that $\mathcal{C}$ is LCD. Let $G^{\prime}$ be a generator matrix of $\mathcal{C}$. Then from Proposition 2.1, $G^{\prime} G^{\prime T}$ is an invertible symmetric matrix of size $k \times k$. Since $\mathcal{C}$ is even-like, all diagonal entries of $G^{\prime} G^{\prime T}$ equal 0 . Then from Part (i) of Proposition 2.2. $k$ is even and there exists nonsingular $k \times k$ matrix $Q$ such that $Q G^{\prime} G^{T} Q^{T}=\left(Q G^{\prime}\right)\left(Q G^{\prime}\right)^{T}=\operatorname{diag}[\underbrace{J_{2}, J_{2}, \ldots, J_{2}}_{\frac{k}{2}}]$, where $J_{2}=\left[\begin{array}{ll}0 & 1 \\ 1 & 0\end{array}\right]$. Let $G=Q G^{\prime}$. Then $G$ is also a generator matrix of $\mathcal{C}$. Let $\mathbf{c}_{i}$ be the $(2 i-1)$-th row of matrix $G$ and $\mathbf{c}_{i}^{\prime}$ be the $2 i$-th row of matrix $G$ for $i \in\left\{1,2, \ldots, \frac{k}{2}\right\}$. Hence $\mathbf{c}_{1}, \mathbf{c}_{1}^{\prime}, \mathbf{c}_{2}, \mathbf{c}_{2}^{\prime}, \ldots, \mathbf{c}_{\frac{k}{2}}, \mathbf{c}_{\frac{k}{2}}^{\prime}$ is the desired basis, because $G G^{T}=\operatorname{diag}[\underbrace{J_{2}, J_{2}, \ldots, J_{2}}_{\frac{k}{2}}]$. It completes the proof.

Remark 2: A basis of $\mathcal{C}$ satisfying the conditions (i), (ii), and (iii) in Theorem 3.2 is called a symplectic basis. Theorem 3.2 shows that an even-like code is LCD if and only if it has a symplectic basis.

Let $d_{\mathrm{LCD}}(n, k)$ be the maximum of possible values of $d$ among $[n, k, d]$ binary LCD codes. Dougherty et al. [6] gave a linear programming bound on the largest size of $d_{\mathrm{LCD}}(n, k)$. Using the idea of principal submatrices, Galvez et al. [7] proved that $d_{\mathrm{LCD}}(n, k) \leq d_{\mathrm{LCD}}(n, k-1)$ if $k$ is odd and $d_{\mathrm{LCD}}(n, k) \leq d_{\mathrm{LCD}}(n, k-2)$ if $k$ is even. They also conjectured that $d_{\mathrm{LCD}}(n, k) \leq d_{\mathrm{LCD}}(n, k-1)$ for any $k$. We will prove this conjecture using the new characterization of binary LCD codes described above. To this end, the following lemma is needed.

Lemma 3.3: Let $\mathcal{C}$ be an even-like binary LCD code with parameters $[n, k]$. Then, there exists a basis $\mathbf{c}_{1}, \mathbf{c}_{1}^{\prime}, \mathbf{c}_{2}, \mathbf{c}_{2}^{\prime}, \ldots, \mathbf{c}_{\frac{k}{2}}, \mathbf{c}_{\frac{k}{2}}^{\prime}$ of $\mathcal{C}$ such that for any $i, j \in\left\{1,2, \ldots, \frac{k}{2}\right\}$, the following conditions hold

(i) $\mathbf{c}_{i} \cdot \mathbf{c}_{j}=\mathbf{c}_{i}^{\prime} \cdot \mathbf{c}_{j}^{\prime}=0$;

(ii) $\mathbf{c}_{i} \cdot \mathbf{c}_{j}^{\prime}=0$ if $i \neq j$;

(iii) $\mathbf{c}_{i} \cdot \mathbf{c}_{i}^{\prime}=1$;

(iv) $c_{i, 1}=c_{i, 1}^{\prime}$, where $\mathbf{c}_{i}=\left(c_{i, 1}, \ldots, c_{i, n}\right)$ and $\mathbf{c}_{i}^{\prime}=\left(c_{i, 1}^{\prime}, \ldots, c_{i, n}^{\prime}\right)$.

Proof From Theorem 3.2, there exists a basis $\mathbf{c}_{1}, \mathbf{c}_{1}^{\prime}, \mathbf{c}_{2}, \mathbf{c}_{2}^{\prime}, \ldots, \mathbf{c}_{\frac{k}{2}}, \mathbf{c}_{\frac{k}{2}}^{\prime}$ of $\mathcal{C}$, which satisfy the conditions (i), (ii) and (iii).

Without loss of generality, assume that $c_{i, 1}=c_{i, 1}^{\prime}+1=1$ for $1 \leq i \leq l$ and $c_{i, 1}=c_{i, 1}^{\prime}$ for $l+1 \leq i \leq k$, where $l$ is some positive integer, $\mathbf{c}_{i}=\left(c_{i, 1}, \ldots, c_{i, n}\right)$, and $\mathbf{c}_{i}^{\prime}=\left(c_{i, 1}^{\prime}, \ldots, c_{i, n}^{\prime}\right)$. Let $\mathbf{w}_{i}=\mathbf{c}_{i}$ for $i \in\left\{1, \ldots, \frac{k}{2}\right\}, \mathbf{w}_{i}^{\prime}=\mathbf{c}_{i}^{\prime}+\mathbf{c}_{i}$ for $i \in\{1, \ldots, l\}$ and $\mathbf{w}_{i}^{\prime}=\mathbf{c}_{i}^{\prime}$ for $i \in\left\{l+1, \ldots, \frac{k}{2}\right\}$. It can be verified directly that the basis $\mathbf{w}_{1}, \mathbf{w}_{1}^{\prime}, \mathbf{w}_{2}, \mathbf{w}_{2}^{\prime}, \ldots, \mathbf{w}_{\frac{k}{2}}, \mathbf{w}_{\frac{k}{2}}^{\prime}$ satisfy the conditions (i), (ii), (iii) and (iv).

Theorem 3.4: If $2 \leq k \leq n$, then $d_{\mathrm{LCD}}(n, k) \leq d_{\mathrm{LCD}}(n, k-1)$.

Proof Let $k$ be odd and $\mathcal{C}$ be an $[n, k]$ LCD code with minimum distance $d=d_{\mathrm{LCD}}(n, k)$. Then, $\mathcal{C}$ is odd-like. From Theorem 3.1 there exists a basis $\mathbf{c}_{1}, \ldots, \mathbf{c}_{k}$ of $\mathcal{C}$ such that $\mathbf{c}_{i} \cdot \mathbf{c}_{j}=1$ if $i=j$ and $\mathbf{c}_{i} \cdot \mathbf{c}_{j}=0$ otherwise. From Theorem 3.1 the code $\mathcal{C}^{\prime}=\operatorname{Span}\left\{\mathbf{c}_{1}, \ldots, \mathbf{c}_{k-1}\right\}$ is an $[n, k-1] \operatorname{LCD}$ code with minimum distance at least $d_{\mathrm{LCD}}(n, k)$.

Let $k$ be even and $\mathcal{C}$ be an $[n, k]$ LCD code with minimum distance $d=d_{\mathrm{LCD}}(n, k)$. If $\mathcal{C}$ is odd-like, the results follow from a similar discussion in the case $k$ odd.

In the following, assume that $\mathcal{C}$ is even-like. If for any $\mathbf{c}=\left(c_{1}, \ldots, c_{n}\right) \in \mathcal{C}, c_{1}=0$. Let $\mathbf{c}_{1}, \mathbf{c}_{1}^{\prime}, \ldots, \mathbf{c}_{\frac{k}{2}}, \mathbf{c}_{\frac{k}{2}}^{\prime}$ be a basis of $\mathcal{C}$ satisfying the conditions in Theorem 3.2 and $\mathcal{C}^{\prime}=\operatorname{Span}\left\{\mathbf{c}_{1}, \mathbf{c}_{1}^{\prime}, \ldots, \mathbf{c}_{\frac{k}{2}-1}, \mathbf{c}_{\frac{k}{2}-1}^{\prime}, \mathbf{w}_{\frac{k}{2}}\right\}$, where $\mathbf{w}_{\frac{k}{2}}=\mathbf{c}_{\frac{k}{2}}+\mathbf{e}_{1}$. Then, $\mathcal{C}^{\prime}$ is an $[n, k-1]$ code with minimum distance at least $d_{\mathrm{LCD}}(n, k)$. Let $G^{\prime}$ be the generator matrix corresponding to the basis $\mathbf{c}_{1}, \mathbf{c}_{1}^{\prime}, \ldots, \mathbf{c}_{\frac{k}{2}-1}, \mathbf{c}_{\frac{k}{2}-1}^{\prime}, \mathbf{w}_{\frac{k}{2}}$. Then, $G^{\prime} G^{\prime T}=\operatorname{diag}[\underbrace{J_{2}, J_{2}, \ldots, J_{2}}_{\frac{k}{2}-1}, 1]$, where $J_{2}=\left[\begin{array}{ll}0 & 1 \\ 1 & 0\end{array}\right]$. It is observed that $G^{\prime} G^{\prime T}$ is nonsingular. By Proposition 2.1 $\mathcal{C}^{\prime}$ is LCD. Thus, the results hold.

If for some $\mathbf{c}=\left(c_{1}, \ldots, c_{n}\right) \in \mathcal{C}, c_{1} \neq 0$. Then, there exists a basis $\mathbf{c}_{1}, \mathbf{c}_{1}^{\prime}, \mathbf{c}_{2}, \mathbf{c}_{2}^{\prime}, \ldots, \mathbf{c}_{\frac{k}{2}}, \mathbf{c}_{\frac{k}{2}}^{\prime}$ of $\mathcal{C}$ satisfying the conditions in Lemma 3.3. Without loss of generality, assume that $c_{i, 1}=c_{i, 1}^{\prime}=1$ for $i \in\{1, \ldots, l\}$ and $c_{i, 1}=c_{i, 1}^{\prime}=0$ for $i \in\left\{l+1, \ldots, \frac{k}{2}\right\}$, where $l$ is some positive integer. Let $\mathcal{C}^{\prime}=\operatorname{Span}\left\{\mathbf{c}_{1}^{\prime}+\mathbf{c}_{1}+\mathbf{e}_{1}\right\}+\operatorname{Span}\left\{\mathbf{c}_{i}+\mathbf{c}_{1}, \mathbf{c}_{i}^{\prime}+\mathbf{c}_{1}: 2 \leq i \leq l\right\}+\operatorname{Span}\left\{\mathbf{c}_{i}, \mathbf{c}_{i}^{\prime}: l+1 \leq i \leq \frac{k}{2}\right\}$. 
Then, $\mathcal{C}^{\prime}$ is an $[n, k-1]$ code with minimum distance at least $d_{\mathrm{LCD}}(n, k)$. Let $G^{\prime}$ be the generator matrix corresponding to the basis $\left\{\mathbf{c}_{1}^{\prime}+\mathbf{c}_{1}+\mathbf{e}_{1}\right\} \cup\left\{\mathbf{c}_{i}+\mathbf{c}_{1}, \mathbf{c}_{i}^{\prime}+\mathbf{c}_{1}: 2 \leq i \leq l\right\} \cup\left\{\mathbf{c}_{i}, \mathbf{c}_{i}^{\prime}: l+1 \leq i \leq \frac{k}{2}\right\}$. It is observed that, for $2 \leq i \leq l$,

$$
\left(\mathbf{c}_{1}^{\prime}+\mathbf{c}_{1}+\mathbf{e}_{1}\right) \cdot\left(\mathbf{c}_{i}+\mathbf{c}_{1}\right)=\left(\mathbf{c}_{1}^{\prime}+\mathbf{c}_{1}+\mathbf{e}_{1}\right) \cdot\left(\mathbf{c}_{i}^{\prime}+\mathbf{c}_{1}\right)=1 \text {, }
$$

for $l \leq i \leq \frac{k}{2}$

$$
\left(\mathbf{c}_{1}^{\prime}+\mathbf{c}_{1}+\mathbf{e}_{1}\right) \cdot \mathbf{c}_{i}=\left(\mathbf{c}_{1}^{\prime}+\mathbf{c}_{1}+\mathbf{e}_{1}\right) \cdot \mathbf{c}_{i}^{\prime}=0,
$$

and $\left(\mathbf{c}_{1}^{\prime}+\mathbf{c}_{1}+\mathbf{e}_{1}\right) \cdot\left(\mathbf{c}_{1}^{\prime}+\mathbf{c}_{1}+\mathbf{e}_{1}\right)=1$. Then,

$$
G^{\prime} G^{\prime T}=\left[\begin{array}{cc}
1 & \mathbf{u} \\
\mathbf{u}^{T} & \operatorname{diag}[\underbrace{J_{2}, J_{2}, \ldots, J_{2}}_{\frac{k}{2}-1}]
\end{array}\right],
$$

where $\mathbf{u}=(\underbrace{1, \ldots, 1}_{2(l-1)}, 0, \ldots, 0) \in \mathbb{F}_{2}^{k-2}$. That is, $G^{\prime} G^{\prime T}$ is a matrix of the following form

$$
\left[\begin{array}{ccccccccc}
1 & 1 & 1 & \ldots & 1 & 1 & 0 & 0 \ldots 0 & 0 \\
1 & 0 & 1 & \ldots & 0 & 0 & 0 & 0 \ldots 0 & 0 \\
1 & 1 & 0 & \ldots & 0 & 0 & 0 & 0 \ldots 0 & 0 \\
\vdots & \vdots & \vdots & \ldots & \vdots & \vdots & \vdots & \vdots \ldots & \vdots \\
1 & 0 & 0 & \ldots & 0 & 1 & 0 & 0 \ldots 0 & 0 \\
1 & 0 & 0 & \ldots & 1 & 0 & 0 & 0 \ldots 0 & 0 \\
0 & 0 & 0 & \ldots & 0 & 0 & 0 & 1 \ldots 0 & 0 \\
0 & 0 & 0 & \ldots & 0 & 0 & 1 & 0 \ldots 0 & 0 \\
\vdots & \vdots & \vdots & \ldots & \vdots & \vdots & \vdots & \vdots \ldots & \vdots \\
0 & 0 & 0 & \ldots & 0 & 0 & 0 & 0 \ldots 0 & 1 \\
0 & 0 & 0 & \ldots & 0 & 0 & 0 & 0 \ldots 1 & 0
\end{array}\right]
$$

Adding the 2-th column, 3-th column, ...,(2l-1)-th column of Matrix (1) to the first column of Matrix (1), one has

$$
\left[\begin{array}{ccccccccc}
1 & 1 & 1 & \ldots & 1 & 1 & 0 & 0 \ldots 0 & 0 \\
0 & 0 & 1 & \ldots & 0 & 0 & 0 & 0 \ldots 0 & 0 \\
0 & 1 & 0 & \ldots & 0 & 0 & 0 & 0 \ldots 0 & 0 \\
\vdots & \vdots & \vdots & \ldots & \vdots & \vdots & \vdots & \vdots \ldots & \vdots \\
0 & 0 & 0 & \ldots & 0 & 1 & 0 & 0 \ldots 0 & 0 \\
0 & 0 & 0 & \ldots & 1 & 0 & 0 & 0 \ldots 0 & 0 \\
0 & 0 & 0 & \ldots & 0 & 0 & 0 & 1 \ldots 0 & 0 \\
0 & 0 & 0 & \ldots & 0 & 0 & 1 & 0 \ldots 0 & 0 \\
\vdots & \vdots & \vdots & \ldots & \vdots & \vdots & \vdots & \vdots & \vdots \\
0 & 0 & 0 & \ldots & 0 & 0 & 0 & 0 \ldots 0 & \vdots \\
0 & 0 & 0 & \ldots & 0 & 0 & 0 & 0 \ldots 1 & 0
\end{array}\right],
$$

which is nonsingular. From Proposition 2.1, $\mathcal{C}^{\prime}$ is LCD, which completes the proof.

\section{THE ACTION OF THE ORTHOGONAL GROUP ON THE SET OF BINARY LCD CODES}

In this section, we will consider the action of the orthogonal group on the set of binary LCD codes.

The set of all binary $\operatorname{LCD}$ codes with parameters $[n, k]$ is denoted by $\operatorname{LCD}[n, k]$. Let $\operatorname{LCD}_{o, o}[n, k]\left(\operatorname{LCD}_{o, e}[n, k]\right.$, respectively) be the set of all odd-like binary LCD codes $\mathcal{C}$ of length $n$ and dimension $k$ such that $\mathcal{C}^{\perp}$ is odd-like (even-like, respectively). We can define $\mathrm{LCD}_{e, o}[n, k]$ and $\mathrm{LCD}_{e, e}[n, k]$ similarly. Obviously there is no even-like binary LCD code with even-like dual and $\operatorname{LCD}_{e, e}[n, k]$ is an empty set. Thus,

$$
\operatorname{LCD}[n, k]=\operatorname{LCD}_{o, o}[n, k] \cup \operatorname{LCD}_{o, e}[n, k] \cup \operatorname{LCD}_{e, o}[n, k] .
$$

Further, the mapping $\mathcal{C} \rightarrow \mathcal{C}^{\perp}$ gives a one-to-one correspondence between $\operatorname{LCD}_{o, e}[n, k]$ and $\operatorname{LCD}_{e, o}[n, n-k]$. We shall say that two binary $\mathrm{LCD}$ codes $\mathcal{C}$ and $\mathcal{C}^{\prime}$ have the same type if they are both in $\operatorname{LCD}_{o, o}[n, k]$, or $\operatorname{LCD}_{o, e}[n, k]$, or $\operatorname{LCD}_{e, o}[n, k]$. 
For any $\mathbf{v}_{1}, \ldots, \mathbf{v}_{k} \in \mathbb{F}_{2}^{n}, \operatorname{Span}\left\{\mathbf{v}_{1}, \ldots, \mathbf{v}_{k}\right\}$ denotes the linear subspace of $\mathbb{F}_{2}^{n}$ spanned by $\mathbf{v}_{1}, \ldots, \mathbf{v}_{k}$. Let $\mathbf{e}_{i}=(0, \ldots, 1, \ldots, 0) \in$ $\mathbb{F}_{2}^{n}$ be the vector with 1 in the $i$-th position. We define the following three matrices:

$$
G_{o, o}=\left[\begin{array}{c}
\mathbf{e}_{1} \\
\mathbf{e}_{2} \\
\mathbf{e}_{3} \\
\mathbf{e}_{4} \\
\vdots \\
\mathbf{e}_{k-1} \\
\mathbf{e}_{k}
\end{array}\right], G_{o, e}=\left[\begin{array}{c}
\sum_{i=1}^{n-k+1} \mathbf{e}_{i} \\
\mathbf{e}_{n-k+2} \\
\mathbf{e}_{n-k+3} \\
\mathbf{e}_{n-k+4} \\
\vdots \\
\mathbf{e}_{n-1} \\
\mathbf{e}_{n}
\end{array}\right] \text { and } G_{e, o}=\left[\begin{array}{c}
\mathbf{e}_{1}+\mathbf{e}_{2} \\
\mathbf{e}_{2}+\mathbf{e}_{3} \\
\mathbf{e}_{1}+\mathbf{e}_{2}+\mathbf{e}_{3}+\mathbf{e}_{4} \\
\mathbf{e}_{4}+\mathbf{e}_{5} \\
\vdots \\
\sum_{i=1}^{k-1} \mathbf{e}_{i}+\mathbf{e}_{k} \\
\mathbf{e}_{k}+\mathbf{e}_{k+1}
\end{array}\right] \text {. }
$$

Let $\mathcal{C}_{o, o}, \mathcal{C}_{o, e}$ and $\mathcal{C}_{e, o}$ denote the linear codes generated by the matrices $G_{o, o}, G_{o, e}$ and $G_{e, o}(k$ even), respectively.

Let $0<k<n$. From Theorem 3.2, if $k\left(n-k\right.$, respectively) is odd, $\operatorname{LCD}_{e, o}[n, k]\left(\operatorname{LCD}_{o, e}[n, k]\right.$, respectively) is empty. The following proposition shows that if $k\left(n-k\right.$, respectively) is even, $\mathrm{LCD}_{e, o}[n, k]$ ( $\mathrm{LCD}_{o, e}[n, k]$, respectively) is non empty.

Proposition 4.1: Let $0<k<n$. Let $\mathcal{C}_{o, o}, \mathcal{C}_{o, e}$ and $\mathcal{C}_{e, o}$ be defined as above. Then, $\mathcal{C}_{o, o} \in \mathrm{LCD}_{o, o}[n, k]$. Moreover, if $k$ ( $n-k$, respectively) is even, $\mathcal{C}_{e, o} \in \mathrm{LCD}_{e, o}[n, k]\left(\mathcal{C}_{o, e} \in \mathrm{LCD}_{o, e}[n, k]\right.$, respectively).

Proof It is easy to verify that $\mathcal{C}_{o, o}^{\perp}=\operatorname{Span}\left\{\mathbf{e}_{i}: i \in\{k+1, \ldots, n\}\right\}$. Then, $\mathcal{C}_{o, o} \cap \mathcal{C}_{o, o}^{\perp}=\{\mathbf{0}\}$ and $\mathcal{C}_{o, o} \in \operatorname{LCD}_{o, o}[n, k]$.

It is observed that

$$
\mathcal{C}_{e, o}^{\perp}=\operatorname{Span}\left\{\sum_{i=1}^{k+1} \mathbf{e}_{i}, \mathbf{e}_{k+2}, \ldots, \mathbf{e}_{n}\right\}
$$

and

$$
\mathcal{C}_{o, e}^{\perp}=\operatorname{Span}\left\{\mathbf{e}_{i}+\mathbf{e}_{n-k+1}: i \in\{1,2, \ldots, n-k\}\right\} .
$$

Then, if $k$ ( $n-k$, respectively) is even, $\mathcal{C}_{e, o} \cap \mathcal{C}_{e, o}^{\perp}=\{\mathbf{0}\}\left(\mathcal{C}_{e, o} \cap \mathcal{C}_{e, o}^{\perp}=\{\mathbf{0}\}\right.$, respectively), which completes the proof.

Remark 3: Let $H_{o, o}, H_{o, e}$ and $H_{e, o}$ be matrices defined by

$$
H_{o, o}=\left[\begin{array}{c}
\mathbf{e}_{k+1} \\
\mathbf{e}_{k+2} \\
\mathbf{e}_{k+3} \\
\mathbf{e}_{k+4} \\
\vdots \\
\mathbf{e}_{n-1} \\
\mathbf{e}_{n}
\end{array}\right], H_{o, e}=\left[\begin{array}{c}
\sum_{i=1}^{1} \mathbf{e}_{i}+\mathbf{e}_{2} \\
\mathbf{e}_{2}+\mathbf{e}_{3} \\
\sum_{i=1}^{3} \mathbf{e}_{i}+\mathbf{e}_{4} \\
\mathbf{e}_{4}+\mathbf{e}_{5} \\
\vdots \\
\sum_{i=1}^{n-k-1} \mathbf{e}_{i}+\mathbf{e}_{n-k} \\
\mathbf{e}_{n-k}+\mathbf{e}_{n-k+1}
\end{array}\right] \text { and } H_{e, o}=\left[\begin{array}{c}
\sum_{i=1}^{k+1} \mathbf{e}_{i} \\
\mathbf{e}_{k+2} \\
\mathbf{e}_{k+3} \\
\mathbf{e}_{k+4} \\
\vdots \\
\mathbf{e}_{n-1} \\
\mathbf{e}_{n}
\end{array}\right] .
$$

Then, from the proof of Proposition 4.1 $H_{o, o}, H_{o, e}$ and $H_{e, o}$ are parity-check matrices of $\mathcal{C}_{o, o}, \mathcal{C}_{o, e}$ and $\mathcal{C}_{e, o}$ respectively.

For any $[n, k]$ linear code $\mathcal{C}$ and $n \times n$ matrix $Q$, let $\mathcal{C} Q$ be the linear code defined by

$$
\mathcal{C} Q=\{\mathbf{c} Q: \mathbf{c} \in \mathcal{C}\} .
$$

In the rest of the paper, $\mathbb{G L}_{n}$ denotes the general linear group of degree $n$ over $\mathbb{F}_{2}$, which is the set of $n \times n$ invertible matrices over $\mathbb{F}_{2}$, together with the operation of ordinary matrix multiplication. A binary orthogonal matrix or orthogonal matrix is a square matrix with binary entries whose columns and rows are orthogonal unit vectors (i.e., orthonormal vectors), i.e. $Q^{T} Q=Q Q^{T}=I$, where $I$ is the identity matrix. The set of $n \times n$ orthogonal matrices forms a group $\mathbb{O}_{n}$, known as the orthogonal group. Recall that an $n \times n$ matrix $Q$ is an orthogonal matrix if and only if $(\mathbf{u} Q) \cdot(\mathbf{v} Q)=\mathbf{u} \cdot \mathbf{v}$ for any $\mathbf{u}, \mathbf{v} \in \mathbb{F}_{2}^{n}$.

Theorem 4.2: Let $\mathcal{C}_{1}$ and $\mathcal{C}_{2}$ be two binary $[n, k]$ LCD codes of the same type. Then, there exists an orthogonal matrix $Q \in \mathbb{O}_{n}$ such that $\mathcal{C}_{2}=\mathcal{C}_{1} Q$. Conversely, for any binary $[n, k]$ LCD code $\mathcal{C}$ and any orthogonal matrix $Q \in \mathbb{O}_{n}, \mathcal{C} Q$ is also an LCD code with the same type as $\mathcal{C}$.

Proof We first consider the case $\mathcal{C}_{1}, \mathcal{C}_{2} \in \mathrm{LCD}_{o, o}[n, k]$. Then, $\mathcal{C}_{1}$ and $\mathcal{C}_{1}^{\perp}$ are odd-like LCD codes. From Theorem 3.1 , there is an orthonormal basis $\mathbf{c}_{1}, \ldots, \mathbf{c}_{k}$ of $\mathcal{C}_{1}$ and an orthonormal basis $\mathbf{c}_{k+1}, \ldots, \mathbf{c}_{n}$ of $\mathcal{C}_{1}^{\perp}$. Then, $\mathbf{c}_{1}, \ldots, \mathbf{c}_{n}$ is an orthonormal basis of $C_{1} \oplus C_{1}^{\perp}=\mathbb{F}_{2}^{n}$. Similarly, there is another orthonormal basis $\mathbf{w}_{1}, \ldots, \mathbf{w}_{n}$ of $\mathbb{F}_{2}^{n}$ such that $\mathcal{C}_{2}=\operatorname{Span}\left\{\mathbf{w}_{1}, \ldots, \mathbf{w}_{k}\right\}$ and $\mathcal{C}_{2}^{\perp}=\operatorname{Span}\left\{\mathbf{w}_{k+1}, \ldots, \mathbf{w}_{n}\right\}$. Let $Q_{1}$ and $Q_{2}$ be orthogonal matrices defined by

$$
Q_{1}=\left[\begin{array}{c}
\mathbf{c}_{1} \\
\mathbf{c}_{2} \\
\vdots \\
\mathbf{c}_{n}
\end{array}\right] \text { and } Q_{2}=\left[\begin{array}{c}
\mathbf{w}_{1} \\
\mathbf{w}_{2} \\
\vdots \\
\mathbf{w}_{n}
\end{array}\right]
$$


Let $Q=Q_{1}^{-1} Q_{2}$. Then, $Q$ is an orthogonal matrix and $\mathbf{c}_{i} Q=\mathbf{w}_{i}$ for $i \in\{1,2, \ldots, k\}$. Thus, $\mathcal{C}_{1} Q=\mathcal{C}_{2}$.

If $\mathcal{C}_{1}, \mathcal{C}_{2} \in \mathrm{LCD}_{o, e}[n, k], \mathcal{C}_{1}$ is an odd-like LCD code and $\mathcal{C}_{1}^{\perp}$ is even-like LCD code. By Theorem 3.1 there is an orthonormal basis $c_{1}, \ldots, c_{k}$ of $\mathcal{C}_{1}$. From Theorem 3.2, there is a basis $\mathbf{c}_{k+1}, \mathbf{c}_{k+1}^{\prime}, \ldots, \mathbf{c}_{k+\frac{n-k}{2}}, \mathbf{c}_{k+\frac{n-k}{2}}^{\prime}$ of $\mathcal{C}_{1}^{\perp}$, which satisfies the conditions (i), (ii) and (iii) in Theorem 3.2. Similarly, $\mathcal{C}_{2}$ has an orthonormal basis $\mathbf{w}_{1}, \ldots, \mathbf{w}_{k}$ and $\mathcal{C}_{2}^{\perp}$ has a basis $\mathbf{w}_{k+1}, \mathbf{w}_{k+1}^{\prime}, \ldots, \mathbf{w}_{k+\frac{n-k}{2}}, \mathbf{w}_{k+\frac{n-k}{2}}^{\prime}$, which satisfies the conditions (i), (ii) and (iii) in Theorem 3.2 Let $Q_{1}$ and $Q_{2}$ be matrices defined by

$$
Q_{1}=\left[\begin{array}{c}
\mathbf{c}_{1} \\
\vdots \\
\mathbf{c}_{k} \\
\mathbf{c}_{k+1} \\
\mathbf{c}_{k+1}^{\prime} \\
\vdots \\
\mathbf{c}_{k+\frac{n-k}{2}} \\
\mathbf{c}_{k+\frac{n-k}{2}}^{\prime}
\end{array}\right] \text { and } Q_{2}=\left[\begin{array}{c}
\mathbf{w}_{1} \\
\vdots \\
\mathbf{w}_{k} \\
\mathbf{w}_{k+1} \\
\mathbf{w}_{k+1}^{\prime} \\
\vdots \\
\mathbf{w}_{k+\frac{n-k}{2}} \\
\mathbf{w}_{k+\frac{n-k}{2}}^{\prime}
\end{array}\right]
$$

Then,

$$
Q_{1} Q_{1}^{T}=Q_{2} Q_{2}^{T}=\left[\begin{array}{cc}
I_{k} & 0 \\
0 & \operatorname{diag}[\underbrace{J_{2}, \ldots, J_{2}}_{\frac{n-k}{2}}]
\end{array}\right]
$$

Let $Q=Q_{1}^{-1} Q_{2}$. One obtains $Q Q^{T}=Q_{1}^{-1}\left(Q_{2} Q_{2}^{T}\right)\left(Q_{1}^{-1}\right)^{T}=Q_{1}^{-1}\left(Q_{1} Q_{1}^{T}\right)\left(Q_{1}^{-1}\right)^{T}=I_{n}$, that is, $Q$ is an orthogonal matrix. It is observed that $\mathbf{c}_{i} Q=\mathbf{w}_{i}$ for every $i \in\{1, \ldots, k\}$. Thus, $\mathcal{C}_{1} Q=\mathcal{C}_{2}$.

If $\mathcal{C}_{1}, \mathcal{C}_{2} \in \operatorname{LCD}_{e, o}[n, k]$, by a similar argument as the case $\mathcal{C}_{1}, \mathcal{C}_{2} \in \operatorname{LCD}_{o, e}[n, k]$, one can prove that there is a $Q \in \mathbb{O}_{n}$ such that $\mathcal{C}_{2}=\mathcal{C}_{1} Q$. Hence, we prove that if $\mathcal{C}_{1}, \mathcal{C}_{2}$ have the same type, there is always an orthogonal matrix $Q$ such that $\mathcal{C}_{2}=\mathcal{C}_{1} Q$.

Conversely, let $\mathcal{C}$ be an $[n, k]$ LCD code and $Q \in \mathbb{O}_{n}$. Recall that $\operatorname{wt}(\mathbf{v})(\bmod 2) \equiv \mathbf{v} \cdot \mathbf{v}=(\mathbf{v} Q) \cdot(\mathbf{v} Q)$ for any $\mathbf{v} \in \mathbb{F}_{2}^{n}$. Then, $\mathcal{C} Q$ is odd-like (even-like, respectively) if and only if $\mathcal{C}$ is odd-like (even-like, respectively). Let $G$ be a generator matrix of $\mathcal{C}$. Then, $G Q$ is a generator matrix of $\mathcal{C} Q$. Note that $(G Q)(G Q)^{T}=G G^{T}$. Thus, $\mathcal{C} Q$ is LCD and its type is as the same as $\mathcal{C}$, which completes the proof.

Theorem 4.2 shows that the orthogonal group $\mathbb{O}_{n}$ acts on $\operatorname{LCD}[n, k]$ by $(\mathcal{C}, Q) \longmapsto \mathcal{C} Q$, where $\mathcal{C} \in \operatorname{LCD}[n, k]$ and $Q \in \mathbb{O}_{n}$. The following theorem presents the decomposition of $\operatorname{LCD}[n, k]$ into $\mathbb{O}_{n}$-orbits.

Theorem 4.3: Let $k$ and $n$ be two positive integers such that $k<n$.

(i) If $n$ is odd and $k$ is odd, $\operatorname{LCD}[n, k]$ can be decomposed as the following disjoint union of orbits

$$
\operatorname{LCD}[n, k]=\mathcal{C}_{o, o} \mathbb{O}_{n} \cup \mathcal{C}_{o, e} \mathbb{O}_{n} .
$$

(ii) If $n$ is odd and $k$ is even, $\operatorname{LCD}[n, k]$ can be decomposed as the following disjoint union of orbits

$$
\operatorname{LCD}[n, k]=\mathcal{C}_{o, o} \mathbb{O}_{n} \cup \mathcal{C}_{e, o} \mathbb{O}_{n} .
$$

(iii) If $n$ is even and $k$ is odd, $\operatorname{LCD}[n, k]$ can be decomposed as the following disjoint union of orbits

$$
\operatorname{LCD}[n, k]=\mathcal{C}_{o, o} \mathbb{O}_{n} .
$$

(iv) If $n$ is even and $k$ is even, $\operatorname{LCD}[n, k]$ can be decomposed as the following disjoint union of orbits

$$
\operatorname{LCD}[n, k]=\mathcal{C}_{o, o} \mathbb{O}_{n} \cup \mathcal{C}_{o, e} \mathbb{O}_{n} \cup \mathcal{C}_{e, o} \mathbb{O}_{n} .
$$

Proof Firstly, recall that $\mathrm{LCD}_{o, e}[n, k]=\emptyset$ if $n-k$ is odd and $\operatorname{LCD}_{e, o}[n, k]=\emptyset$ if $k$ is odd. Then, the results follow from Equation (2), Proposition 4.1 and Theorem 4.2

To determine the size of the $\mathbb{O}_{n}$-orbit of a code $\mathcal{C}$, we need to the order of the stabilizer of $\mathcal{C}$, which is defined by

$$
\operatorname{St}(\mathcal{C})=\left\{Q \in \mathbb{O}_{n}: \mathcal{C} Q=\mathcal{C}\right\} .
$$

For an even positive integer, $\mathbb{S p}_{k}$ denotes the symplectic group of degree $k$, a $k \times k$ matrix $Q$ is in $\mathbb{S p}_{k}$ if and only if

$$
Q \operatorname{diag}[\underbrace{J_{2}, \ldots, J_{2}}_{\frac{k}{2}}] Q^{T}=\operatorname{diag}[\underbrace{J_{2}, \ldots, J_{2}}_{\frac{k}{2}}] .
$$


Lemma 4.4: Let $\mathcal{C}$ be a binary $[n, k]$ LCD code, $G$ be a generator matrix of $\mathcal{C}$ and $H$ be a generator matrix of $\mathcal{C}^{\perp}$. Then, $Q \in \operatorname{St}(\mathcal{C})$ if and only if $Q=\left[\begin{array}{c}G \\ H\end{array}\right]^{-1}\left[\begin{array}{cc}Q_{1} & 0 \\ 0 & Q_{2}\end{array}\right]\left[\begin{array}{c}G \\ H\end{array}\right]$, where $Q_{1} \in \mathbb{G L}_{k}, Q_{2} \in \mathbb{G L}_{n-k}$ such that $Q_{1}\left(G G^{T}\right) Q_{1}^{T}=$ $G G^{T}$ and $Q_{2}\left(H H^{T}\right) Q_{2}^{T}=H H^{T}$.

Proof Let $Q=\left[\begin{array}{c}G \\ H\end{array}\right]^{-1}\left[\begin{array}{cc}Q_{1} & 0 \\ 0 & Q_{2}\end{array}\right]\left[\begin{array}{c}G \\ H\end{array}\right]$ satisfying $Q_{1}\left(G G^{T}\right) Q_{1}^{T}=G G^{T}$ and $Q_{2}\left(H H^{T}\right) Q_{2}^{T}=H H^{T}$. Note that $\left[\begin{array}{l}G \\ H\end{array}\right] Q=\left[\begin{array}{cc}Q_{1} & 0 \\ 0 & Q_{2}\end{array}\right]\left[\begin{array}{c}G \\ H\end{array}\right]=\left[\begin{array}{l}Q_{1} G \\ Q_{2} H\end{array}\right]$. Then, $G Q=Q_{1} G$ and $H Q=Q_{2} H$. Thus, $\mathcal{C} Q=\mathcal{C}$. Note that

$$
\begin{aligned}
& {\left[\begin{array}{l}
G \\
H
\end{array}\right] Q Q^{T}\left[\begin{array}{l}
G \\
H
\end{array}\right]^{T}=\left(\left[\begin{array}{l}
G \\
H
\end{array}\right] Q\right)\left(\left[\begin{array}{l}
G \\
H
\end{array}\right] Q\right)^{T}} \\
& =\left[\begin{array}{c}
Q_{1} G \\
Q_{2} H
\end{array}\right]\left[\begin{array}{ll}
G^{T} Q_{1}^{T} & H^{T} Q_{2}^{T}
\end{array}\right] \\
& =\left[\begin{array}{cc}
Q_{1} G G^{T} Q_{1}^{T} & Q_{1} G H^{T} Q_{2}^{T} \\
Q_{2} H G^{T} Q_{1}^{T} & Q_{2} H H^{T} Q_{2}^{T}
\end{array}\right] \\
& =\left[\begin{array}{cc}
Q_{1} G G^{T} Q_{1}^{T} & 0 \\
0 & Q_{2} H H^{T} Q_{2}^{T}
\end{array}\right] \\
& =\left[\begin{array}{cc}
G G^{T} & 0 \\
0 & H H^{T}
\end{array}\right] \\
& =\left[\begin{array}{l}
G \\
H
\end{array}\right]\left[\begin{array}{l}
G \\
H
\end{array}\right]^{T} \text {. }
\end{aligned}
$$

Then, $Q Q^{T}=I_{n}$, that is $Q \in \mathbb{O}_{n}$. Thus, $Q \in \operatorname{St}(\mathcal{C})$.

Conversely, let $Q \in \operatorname{St}(\mathcal{C})$, that is $\mathcal{C} Q=\mathcal{C}$. Then, $G Q=Q_{1} G$ with $Q_{1} \in \mathbb{G}_{k}$. For any $\mathbf{c} \in \mathcal{C}$ and any w $\in \mathcal{C} \perp$, one has $(\mathbf{c} Q) \cdot(\mathbf{w} Q)=\mathbf{c} \cdot \mathbf{w}=0$ from $Q \in \mathbb{O}_{n}$. Then, $\mathcal{C}^{\perp} Q=\mathcal{C}^{\perp}$. Thus, there exists $Q_{2} \in \mathbb{G L}_{n-k}$ such that $H Q=Q Q_{2} H$. One gets $\left[\begin{array}{l}G \\ H\end{array}\right] Q=\left[\begin{array}{cc}Q_{1} & 0 \\ 0 & Q_{2}\end{array}\right]\left[\begin{array}{c}G \\ H\end{array}\right]$. Then, $Q=\left[\begin{array}{c}G \\ H\end{array}\right]^{-1}\left[\begin{array}{cc}Q_{1} & 0 \\ 0 & Q_{2}\end{array}\right]\left[\begin{array}{c}G \\ H\end{array}\right]$. Since $Q \in \mathbb{O}_{n}$, one has

$$
\begin{aligned}
G G^{T} & =(G Q)(G Q)^{T} \\
& =Q_{1}\left(G G^{T}\right) Q_{1}^{T},
\end{aligned}
$$

and

$$
\begin{aligned}
H H^{T} & =(H Q)(H Q)^{T} \\
& =Q_{2}\left(H H^{T}\right) Q_{2}^{T} .
\end{aligned}
$$

It completes the proof.

Corollary 4.5: Let $k$ and $n$ be two positive integers with $0<k<n$.

(i) Let $\mathcal{C}_{o, o}$ be the LCD code with generator matrix $G_{o, o}$ defined by Equation (3). Then,

$$
\operatorname{St}\left(\mathcal{C}_{o, o}\right)=\left\{\left[\begin{array}{cc}
Q_{1} & 0 \\
0 & Q_{2}
\end{array}\right]: Q_{1} \in \mathbb{O}_{k}, Q_{2} \in \mathbb{O}_{n-k}\right\}
$$

(ii) Assume $(n-k)$ be even. Let $G_{o, e}$ and $H_{o, e}$ be matrices defined by Equations (3) and (4) respectively. Then,

$$
\operatorname{St}\left(\mathcal{C}_{o, e}\right)=\left\{\left[\begin{array}{c}
G_{o, e} \\
H_{o, e}
\end{array}\right]^{-1}\left[\begin{array}{cc}
Q_{1} & 0 \\
0 & Q_{2}
\end{array}\right]\left[\begin{array}{c}
G_{o, e} \\
H_{o, e}
\end{array}\right]: Q_{1} \in \mathbb{O}_{k}, Q_{2} \in \mathbb{S p}_{n-k}\right\} .
$$

(iii) Assume $k$ be even. Let $G_{e, o}$ and $H_{e, o}$ be the matrices defined by Equations (3) and (4) respectively. Then,

$$
\operatorname{St}\left(\mathcal{C}_{e, o}\right)=\left\{\left[\begin{array}{c}
G_{e, o} \\
H_{e, o}
\end{array}\right]^{-1}\left[\begin{array}{cc}
Q_{1} & 0 \\
0 & Q_{2}
\end{array}\right]\left[\begin{array}{c}
G_{e, o} \\
H_{e, o}
\end{array}\right]: Q_{1} \in \mathbb{S p}_{k}, Q_{2} \in \mathbb{O}_{n-k}\right\}
$$

Proof Note that $G_{o, o}, G_{o, e}$ and $G_{e, o}$ are generator matrices of $\mathcal{C}_{o, o}, \mathcal{C}_{o, e}$ and $\mathcal{C}_{e, o}$ respectively, and $H_{o, o}, H_{o, e}$ and $H_{e, o}$ are generator matrices of $\mathcal{C}_{o, o}, \mathcal{C}_{o, e}$ and $\mathcal{C}_{e, o}$ respectively. For any $0<k<n, G_{o, o} G_{o, o}^{T}=I_{k}$ and $H_{o, o} H_{o, o}^{T}=I_{n-k}$. For even $(n-k), G_{o, e} G_{o, e}^{T}=I_{k}$ and $H_{o, e} H_{o, e}^{T}=\operatorname{diag}[\underbrace{J_{2}, \ldots, J_{2}}_{\frac{n-k}{2}}]$. For even $k, G_{e, o} G_{e, o}^{T}=\operatorname{diag}[\underbrace{J_{2}, \ldots, J_{2}}_{\frac{k}{2}}]$ and $H_{e, o} H_{e, o}^{T}=I_{n-k}$.

Then, the results follow from Lemma 4.4 
For a finite set $S,|S|$ denotes the cardinality of $S$. To determine the cardinality of the orbit, we need the following formulas, which can be found in [8] and [12].

$$
\left|\mathbb{O}_{k}\right|= \begin{cases}2^{\frac{k^{2}}{4}} \prod_{i=1}^{\frac{k}{2}-1}\left(2^{2 i}-1\right), & \text { if } k \text { is even, } \\ 2^{\frac{(k-1)^{2}}{4}} \prod_{i=1}^{\frac{k-1}{2}}\left(2^{2 i}-1\right), & \text { if } k \text { is odd }\end{cases}
$$

and, for an even positive integer $k$,

$$
\left|\mathbb{S p}_{k}\right|=2^{\frac{k^{2}}{4}} \prod_{i=1}^{\frac{k}{2}}\left(2^{2 i}-1\right)
$$

For a number $q$ with $q \neq 1$, the Gaussian or $q$-binomial coefficient $\left[\begin{array}{l}n \\ k\end{array}\right]_{q}$ is defined to be

$$
\left[\begin{array}{l}
n \\
k
\end{array}\right]_{q}=\frac{\left(q^{n}-1\right)\left(q^{n-2}-1\right) \cdots\left(q^{n-k+1}-1\right)}{(q-1)\left(q^{2}-1\right) \cdots\left(q^{k}-1\right)} .
$$

The Gaussian coefficients has the same symmetry as that of binomial coefficients

$$
\left[\begin{array}{l}
n \\
k
\end{array}\right]_{q}=\left[\begin{array}{c}
n \\
n-k
\end{array}\right]_{q} .
$$

The number of $k$-dimensional subspaces of an $n$-dimensional vector space over $\mathbb{F}_{q}$ is just $\left[\begin{array}{l}n \\ k\end{array}\right]_{q}$.

Theorem 4.6: Let $k$ and $n$ be positive integers with $k<n$.

(i) Let $\operatorname{LCD}_{o, o}[n, k]$ be the set of odd-like binary $[n, k]$ LCD codes with odd-like duals, then

$$
\left|\mathrm{LCD}_{o, o}[n, k]\right|= \begin{cases}2^{\frac{n k-k^{2}+n-1}{2}\left[\begin{array}{c}
\frac{n}{2}-1 \\
\left.\frac{k-1}{2}\right]
\end{array},\right.} & \text { if } k \text { odd, } n \text { even, } \\
2^{\frac{(n-k)(k-1)}{2}}\left(2^{n-k}-1\right)\left[\frac{\frac{n-1}{2}}{\frac{k-1}{2}}\right], & \text { if } k \text { odd, } n \text { odd, } \\
\left.2^{\frac{k(n-k-1)}{2}}\left(2^{k}-1\right)\left[\frac{n-1}{2}\right]_{4}^{\frac{k}{2}}\right]_{4}, & \text { if } k \text { even, } n \text { odd, } \\
2^{\frac{k(n-k)}{2}}\left(2^{k}-1\right)\left[\begin{array}{c}
\frac{n}{2}-1 \\
\frac{k}{2}
\end{array}\right], & \text { if } k \text { even, } n \text { even. }\end{cases}
$$

(ii) Let $\operatorname{LCD}_{o, e}[n, k]$ be the set of odd-like binary $[n, k]$ LCD codes with even-like duals, then

$$
\left|\mathrm{LCD}_{o, e}[n, k]\right|= \begin{cases}2^{\frac{(k-1)(n-k)}{2}\left[\frac{n-1}{2}\right]}, & \text { if } k \text { odd, } n \text { odd, } \\
2^{\frac{k(n-k)}{2}}\left[\begin{array}{l}
\frac{n}{2}-1 \\
\frac{k}{2}-1
\end{array}\right], & \text { if } k \text { even, } n \text { even, } \\
0, & \text { otherwise. }\end{cases}
$$

(iii) Let $\mathrm{LCD}_{e, o}[n, k]$ be the set of even-like binary $[n, k] \mathrm{LCD}$ codes with odd-like duals, then

$$
\left|\mathrm{LCD}_{e, o}[n, k]\right|= \begin{cases}\left.2^{\frac{k(n-k-1)}{2}}\left[\frac{n-1}{2}\right]_{\frac{k}{2}}\right], & \text { if } k \text { even, } n \text { odd, } \\
2^{\frac{k(n-k)}{2}}\left[\begin{array}{c}
\frac{n}{2}-1 \\
\frac{k}{2}
\end{array}\right], & \text { if } k \text { even, } n \text { even, } \\
0, & \text { otherwise. }\end{cases}
$$

Proof From Proposition 4.1 and Theorem 4.2, $\mathrm{LCD}_{o, o}=\mathcal{C}_{o, o} \mathbb{O}_{n}$. Then,

$$
\left|\mathrm{LCD}_{o, o}\right|=\frac{\left|\mathbb{O}_{n}\right|}{\left|\operatorname{St}\left(\mathcal{C}_{o, o}\right)\right|} \text {. }
$$

From Corollary 4.5, one obtains

$$
\left|\mathrm{LCD}_{o, o}\right|=\frac{\left|\mathbb{O}_{n}\right|}{\left|\mathbb{O}_{k}\right| \cdot\left|\mathbb{O}_{n-k}\right|}
$$

Then, Part (i) follows from Equation (6).

By a similar discussion, one has

$$
\left|\mathrm{LCD}_{o, e}\right|=\frac{\left|\mathbb{O}_{n}\right|}{\left|\mathbb{O}_{k}\right| \cdot\left|\mathrm{Sp}_{n-k}\right|}, \text { if }(n-k) \text { is even, }
$$

and

$$
\left|\operatorname{LCD}_{e, o}\right|=\frac{\left|\mathbb{O}_{n}\right|}{\left|\operatorname{Sp}_{k}\right| \cdot\left|\mathbb{O}_{n-k}\right|}, \text { if } k \text { is even. }
$$


Then, Parts (ii) and (iii) follow from Equations (6) and (7). It completes the proof.

Corollary 4.7: Let $k$ and $n$ be positive integers with $k<n$.

(i) Let $\operatorname{LCD}_{o}[n, k]$ be the set of odd-like binary $[n, k]$ LCD codes, then

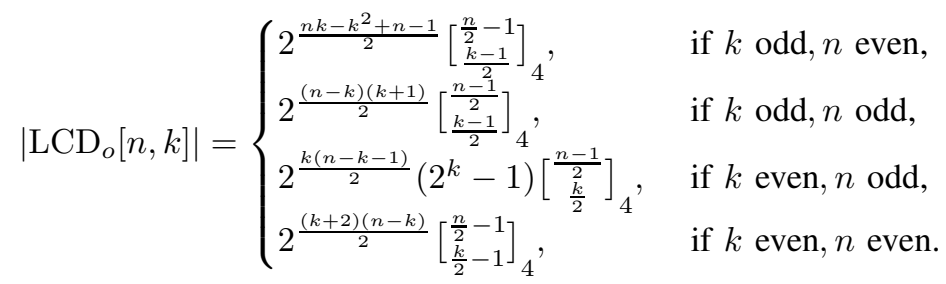

(ii) Let $\operatorname{LCD}_{e}[n, k]$ be the set of even-like binary $[n, k]$ LCD codes, then

$$
\left|\mathrm{LCD}_{e}[n, k]\right|= \begin{cases}\left.2^{\frac{k(n-k-1)}{2}\left[\frac{n-1}{2}\right.}\right]_{\frac{k}{2}}, & \text { if } k \text { even, } n \text { odd, } \\ \left.\left.2^{\frac{k(n-k)}{2}\left[\frac{n}{2}-1\right.}\right]^{\frac{k}{2}}\right]_{4}, & \text { if } k \text { even, } n \text { even, } \\ 0, & \text { otherwise. }\end{cases}
$$

Proof From $\left|\operatorname{LCD}_{o}[n, k]\right|=\left|\mathrm{LCD}_{o, o}[n, k]\right|+\left|\mathrm{LCD}_{o, e}[n, k]\right|$ and Theorem 4.6, Part (i) follows. From $\left|\mathrm{LCD}_{e}[n, k]\right|=$ $\left|\mathrm{LCD}_{e, o}[n, k]\right|$ and Theorem 4.6, Part (ii) follows.

Corollary 4.8: Let $k$ and $n$ be two positive integers with $k<n$. Then,

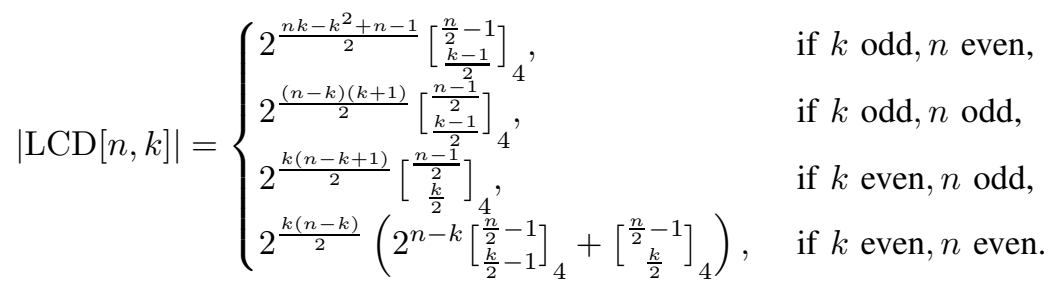

Proof From $|\mathrm{LCD}[n, k]|=\left|\mathrm{LCD}_{o}[n, k]\right|+\left|\mathrm{LCD}_{e}[n, k]\right|$ and Corollary 4.7, this corollary follows.

Remark 4: In [14], Sindrier gave a formula of the number of LCD codes, which involves the number of self-orthogonal codes. Since the number of terms in the summation of the formula is very large, the formula is intractable. The formula we give here is a very simple closed formula.

In the following, we shall analyze the asymptotic behavior of the size of the orbits $\mathcal{C} \mathbb{O}_{n}$. For all $q>1$, let $g_{q, n}$ be the number defined by

$$
g_{q, n}=\prod_{i=1}^{n}\left(1-\frac{1}{q^{i}}\right)
$$

Then, we can rewrite $\left[\begin{array}{l}n \\ k\end{array}\right]_{q}$ as

$$
\left[\begin{array}{l}
n \\
k
\end{array}\right]_{q}=q^{k(n-k)} \frac{g_{q, n}}{g_{q, k} g_{q, n-k}} .
$$

The sequence $g_{q, 1}, g_{q, 2}, \ldots$ is strictly decreasing and has positive limit, which is denoted by $g_{q, \infty}$ or $\prod_{i=1}^{\infty}\left(1-\frac{1}{q^{i}}\right)$. Then, one has

$$
\lim _{\substack{k \rightarrow \infty \\
(n-k) \rightarrow \infty}} \frac{\left[\begin{array}{l}
n \\
k
\end{array}\right]_{q}}{q^{k(n-k)}}=\frac{1}{g_{q, \infty}} .
$$

Theorem 4.9: Let $k$ and $n$ be two positive integers with $k<n$.

(i) Let $\mathrm{LCD}_{o, o}[n, k]$ be the set of odd-like binary $[n, k]$ LCD codes with odd-like duals. Then,

$$
\lim _{\substack{k \rightarrow \infty \\(n-k) \rightarrow \infty}} \frac{\left|\mathrm{LCD}_{o, o}[n, k]\right|}{2^{k(n-k)}}=\frac{1}{g_{4, \infty}} .
$$

(ii) Let $\mathrm{LCD}_{o, e}[n, k]$ be the set of odd-like binary $[n, k] \operatorname{LCD}$ codes with even-like duals. If $(n-k)$ is odd, then $\left|\mathrm{LCD}_{o, e}[n, k]\right|=$ 0 . If $(n-k)$ is even, then

$$
\lim _{\substack{k \rightarrow \infty \\(n-k) \rightarrow \infty \\(n-k) \text { is even }}} \frac{\left|\mathrm{LCD}_{o, e}[n, k]\right|}{2^{(k-1)(n-k)}}=\frac{1}{g_{4, \infty}}
$$


(iii) Let $\mathrm{LCD}_{e, o}[n, k]$ be the set of even-like binary $[n, k] \mathrm{LCD}$ codes with odd-like duals. If $k$ is odd, then $\left|\mathrm{LCD}_{e, o}[n, k]\right|=0$. If $k$ is even, then

$$
\lim _{\substack{k \rightarrow \infty \\(n-k) \rightarrow \infty \\ k \text { is even }}} \frac{\left|\mathrm{LCD}_{e, o}[n, k]\right|}{2^{k(n-k-1)}}=\frac{1}{g_{4, \infty}} .
$$

Proof From Theorem 4.6 and Equation (8), one gets

$$
\left|\mathrm{LCD}_{o, o}[n, k]\right|= \begin{cases}2^{k(n-k)} \frac{g_{4, \frac{n}{2}-1}}{g_{4, \frac{k-1}{2}} g_{4, \frac{n-k-1}{2}}}, & \text { if } k \text { odd, } n \text { even, } \\ 2^{k(n-k)}\left(1-2^{-(n-k)}\right) \frac{g_{4, \frac{n-1}{2}}}{g_{4, \frac{k-1}{2}} g_{4, \frac{n-k}{2}}}, & \text { if } k \text { odd, } n \text { odd, } \\ 2^{k(n-k)}\left(1-2^{-k}\right) \frac{g_{4, \frac{n-1}{2}}}{g_{4, \frac{k}{2}} g_{4, \frac{n-k-1}{2}}}, & \text { if } k \text { even, } n \text { odd, } \\ 2^{k(n-k)}\left(1-2^{-k}\right) \frac{g_{4, \frac{n}{2}-1}}{g_{4, \frac{k}{2}} g_{4, \frac{n-k}{2}}}, & \text { if } k \text { even, } n \text { even. }\end{cases}
$$

From $\lim _{n \rightarrow \infty} g_{4, n}=g_{4, \infty}=\prod_{i=1}^{\infty}\left(1-\frac{1}{2^{2 i}}\right)$, Part (i) follows. By a similar discussion, we can prove that Parts (ii) and (iii) hold. It completes the proof.

Corollary 4.10: Let $k$ and $n$ be two positive integers with $k<n$.

(i) Let $\mathrm{LCD}_{o, o}[n, k]$ be the set of odd-like binary $[n, k]$ LCD codes with odd-like duals. Then,

$$
\lim _{\substack{k \rightarrow \infty \\
(n-k) \rightarrow \infty}} \frac{\left|\mathrm{LCD}_{o, o}[n, k]\right|}{\left[\begin{array}{l}
n \\
k
\end{array}\right]_{2}}=\frac{1}{\prod_{i=1}^{\infty}\left(1+\frac{1}{2^{2}}\right)} .
$$

(ii) Let $\mathrm{LCD}_{o, e}[n, k]$ be the set of odd-like binary $[n, k] \mathrm{LCD}$ codes with even-like duals. If $(n-k)$ is odd, then $\left|\mathrm{LCD}_{o, e}[n, k]\right|=$ 0 . If $(n-k)$ is even, then

$$
\lim _{\substack{k \rightarrow \infty \\
(n-k) \rightarrow \infty \\
(n-k) \text { is even }}} \frac{2^{n-k}\left|\mathrm{LCD}_{o, e}[n, k]\right|}{\left[\begin{array}{l}
n \\
k
\end{array}\right]_{2}}=\frac{1}{\prod_{i=1}^{\infty}\left(1+\frac{1}{2^{i}}\right)} .
$$

(iii) Let $\mathrm{LCD}_{e, o}[n, k]$ be the set of even-like binary $[n, k] \mathrm{LCD}$ codes with odd-like duals. If $k$ is odd, then $\left|\operatorname{LCD}_{e, o}[n, k]\right|=0$. If $k$ is even, then

$$
\lim _{\substack{k \rightarrow \infty \\
n-k \rightarrow \infty \\
k \text { is even }}} \frac{2^{k}\left|\operatorname{LCD}_{e, o}[n, k]\right|}{\left[\begin{array}{l}
n \\
k
\end{array}\right]_{2}}=\frac{1}{\prod_{i=1}^{\infty}\left(1+\frac{1}{2^{i}}\right)} .
$$

Proof From Theorem 4.9 and Equation (9), one obtains

$$
\lim _{\substack{k \rightarrow \infty \\
(n-k) \rightarrow \infty}} \frac{\left|\mathrm{LCD}_{o, o}[n, k]\right|}{\left[\begin{array}{l}
n \\
k
\end{array}\right]_{2}}=\frac{g_{2, \infty}}{g_{4, \infty}} .
$$

It is observed that

$$
\begin{aligned}
\frac{g_{2, \infty}}{g_{4, \infty}} & =\frac{\lim _{n \rightarrow \infty} \prod_{i=1}^{n}\left(1-\frac{1}{2^{i}}\right)}{\lim _{n \rightarrow \infty} \prod_{i=1}^{n}\left(1-\frac{1}{2^{2 i}}\right)} \\
& =\lim _{n \rightarrow \infty} \frac{\prod_{i=1}^{n}\left(1-\frac{1}{2^{i}}\right)}{\prod_{i=1}^{n}\left(1-\frac{1}{2^{2 i}}\right)} \\
& =\lim _{n \rightarrow \infty} \frac{1}{\prod_{i=1}^{n}\left(1+\frac{1}{2^{i}}\right)} \\
& =\frac{1}{\prod_{i=1}^{\infty}\left(1+\frac{1}{2^{i}}\right)} .
\end{aligned}
$$

Then, Part (i) holds. By a similar discussion, we can prove Parts (ii) and (iii).

Corollary 4.11: Let $k$ and $n$ be two positive integers with $k<n$.

(i) Let $\mathrm{LCD}[n, k]$ be the set of binary $[n, k]$ LCD codes. Then,

$$
\lim _{\substack{k \rightarrow \infty \\
(n-k) \rightarrow \infty}} \frac{|\mathrm{LCD}[n, k]|}{\left[\begin{array}{l}
n \\
k
\end{array}\right]_{2}}=\frac{1}{\prod_{i=1}^{\infty}\left(1+\frac{1}{2^{i}}\right)} .
$$


(ii) Let $\mathrm{LCD}_{o, o}[n, k], \mathrm{LCD}_{o, e}[n, k]$ and $\mathrm{LCD}_{e, o}[n, k]$ be defined as in Corollary 4.10 . Then,

$$
\lim _{\substack{k \rightarrow \infty \\(n-k) \rightarrow \infty}} \frac{\left|\mathrm{LCD}_{o, o}[n, k]\right|}{|\mathrm{LCD}[n, k]|}=1
$$

and

$$
\lim _{\substack{k \rightarrow \infty \\(n-k) \rightarrow \infty}} \frac{\left|\mathrm{LCD}_{o, e}[n, k]\right|}{|\mathrm{LCD}[n, k]|}=\frac{\left|\mathrm{LCD}_{e, o}[n, k]\right|}{|\mathrm{LCD}[n, k]|}=0 .
$$

Proof From Corollary 4.10, this corollary follows.

Remark 5: Part (i) of Corollary 4.11 has been proved in [14]. We give a simpler and more direct proof for this result. Part (ii) shows that when $k$ and $(n-k)$ go to infinity, almost all binary LCD codes $\mathcal{C}$ are odd-like codes with odd-like duals $\mathcal{C}^{\perp}$. Accordingly, for almost all binary LCD codes, they and their duals both have orthonormal basis.

In the following, we count the inequivalent binary LCD codes. Let $\mathbb{P}_{n}$ be the group generated by all $n \times n$ permutation matrices, which are square matrices that have exactly one entry of 1 in each row and each column and 0s elsewhere. Two codes $\mathcal{C}_{1}, \mathcal{C}_{2}$ are equivalent if there exists a permutation $P$ in $\mathbb{P}_{n}$ such that $\mathcal{C}_{2}=\mathcal{C}_{1} P$. For every $\mathcal{C} \in \operatorname{LCD}[n, k]$ and every $P \in \mathbb{P}_{n}, \mathcal{C}$ and $\mathcal{C} P$ are in the same orbit. Then, we only need to classify LCD codes over every orbit. We first consider classifying LCD codes in $\operatorname{LCD}_{o, o}[n, k]=\mathcal{C}_{o, o} \mathbb{O}_{n}$. For every $\mathcal{C} \in \operatorname{LCD}[n, k],[\mathcal{C}]$ denotes the equivalence class of $\mathcal{C}$, i.e., $[\mathcal{C}]=\left\{\mathcal{C} P: P \in \mathbb{P}_{n}\right\}$. Let $\widetilde{\operatorname{LCD}}_{o, o}[n, k]=\left\{[\mathcal{C}]: \mathcal{C} \in \operatorname{LCD}_{o, o}[n, k]\right\}$. From $\operatorname{LCD}_{o, o}[n, k]=\mathcal{C}_{o, o} \mathbb{O}_{n}$, there is a one-to-one correspondence between the family $\operatorname{LCD}_{o, o}[n, k]$ of $\mathrm{LCD}$ codes and the the set $\operatorname{St}\left(\mathcal{C}_{o, o}\right) \backslash \mathbb{O}_{n}$ of right cosets defined by

$$
\begin{aligned}
\pi: \mathrm{LCD}_{o, o}[n, k] & \longrightarrow \operatorname{St}\left(\mathcal{C}_{o, o}\right) \backslash \mathbb{O}_{n}, \\
\mathcal{C}_{o, o} Q & \longmapsto \operatorname{St}\left(\mathcal{C}_{o, o}\right) Q .
\end{aligned}
$$

By the definition of $\widetilde{\operatorname{LCD}}_{o, o}[n, k]$, the map $\pi$ induces a surjection $\tilde{\pi}$ between $\widetilde{\operatorname{LCD}}_{o, o}[n, k]$ and the set $\operatorname{St}\left(\mathcal{C}_{o, o}\right) \backslash \mathbb{O}_{n} / \mathbb{P}_{n}$ of double cosets defined as

$$
\begin{aligned}
\tilde{\pi}: \widetilde{\operatorname{LCD}}_{o, o}[n, k] & \longrightarrow \operatorname{St}\left(\mathcal{C}_{o, o}\right) \backslash \mathbb{O}_{n} / \mathbb{P}_{n}, \\
{\left[\mathcal{C}_{o, o} Q\right] } & \longmapsto \operatorname{St}\left(\mathcal{C}_{o, o}\right) Q \mathbb{P}_{n} .
\end{aligned}
$$

Then, we obtain a parametrization of the inequivalent linear codes in $\operatorname{LCD}_{o, o}[n, k]$ by the set $\operatorname{St}\left(\mathcal{C}_{o, o}\right) \backslash \mathbb{O}_{n} / \mathbb{P}_{n}$ of double cosets. Hence, classifying the inequivalent $\mathrm{LCD}$ codes in $\mathrm{LCD}_{o, o}[n, k]$ is equivalent to determining representatives of the set $\operatorname{St}\left(\mathcal{C}_{o, o}\right) \backslash \mathbb{O}_{n} / \mathbb{P}_{n}$ of double cosets.

For any $[n, k]$ linear code $\mathcal{C}$, the automorphism group $\operatorname{Aut}(\mathcal{C})$ of $\mathcal{C}$ is defined by $\operatorname{Aut}(\mathcal{C})=\left\{P \in \mathbb{P}_{n}: \mathcal{C} P=\mathcal{C}\right\}$. Then, we have the mass formula for $\mathrm{LCD}_{o, o}[n, k]$.

Proposition 4.12: Let $k$ and $n$ be two positive integers with $k<n$. Then,

$$
\sum_{[\mathcal{C}] \in \widehat{\operatorname{LCD}}_{o, o}[n, k]} \frac{1}{|\operatorname{Aut}(\mathcal{C})|}=\frac{\left|\mathbb{O}_{n}\right|}{\left|\operatorname{St}\left(\mathcal{C}_{o, o}\right)\right| \cdot\left|\mathbb{P}_{n}\right|} .
$$

Proof From $\operatorname{LCD}_{o, o}[n, k]=\mathcal{C}_{o, o} \mathbb{O}_{n}$, one has

$$
\begin{aligned}
\frac{\left|\mathbb{O}_{n}\right|}{\left|\operatorname{St}\left(\mathcal{C}_{o, o}\right)\right|} & =\sum_{\mathcal{C} \in \operatorname{LCD}_{o, o}[n, k]} 1 \\
& =\sum_{[\mathcal{C}] \in \overbrace{\operatorname{LCD}}^{o, o}[n, k]}|[\mathcal{C}]| \\
& =\sum_{[\mathcal{C}] \in \overbrace{\operatorname{LCD}}^{o, o}[n, k]}\left|\mathcal{C} \mathbb{P}_{n}\right| \\
& =\sum_{[\mathcal{C}] \in \widehat{\operatorname{LCD}}_{o, o}[n, k]} \frac{\left|\mathbb{P}_{n}\right|}{|\operatorname{Aut}(\mathcal{C})|},
\end{aligned}
$$

which completes the proof.

We can consider the problem of classifying the inequivalent $\mathrm{LCD}$ codes in $\operatorname{LCD}_{o, e}[n, k]$ and $\operatorname{LCD}_{e, o}[n, k]$ exactly like the case $\mathrm{LCD}_{o, o}[n, k]$. In short, classifying the inequivalent binary LCD codes is equivalent to determining representatives of the following three sets of double cosets:

$$
\operatorname{St}\left(\mathcal{C}_{o, o}\right) \backslash \mathbb{O}_{n} / \mathbb{P}_{n}, \operatorname{St}\left(\mathcal{C}_{o, e}\right) \backslash \mathbb{O}_{n} / \mathbb{P}_{n}
$$

and

$$
\operatorname{St}\left(\mathcal{C}_{e, o}\right) \backslash \mathbb{O}_{n} / \mathbb{P}_{n}
$$




\section{THE CHARACTERIZATION OF LCD CODES IN ODD CHARACTERISTIC}

In this section, we will consider LCD codes over $\mathbb{F}_{q}$, where $q$ is a power of an odd prime.

The following proposition [15] is very important for the characterization of LCD codes over finite fields of odd characteristic.

Proposition 5.1: If $M$ is a $k \times k$ nonsingular symmetric matrix over $\mathbb{F}_{q}$ with $k \geq 2$, then there is a $k \times k$ nonsingular matrix $Q$ such that $Q M Q^{T}=\operatorname{diag}[\underbrace{1,1, \ldots, 1}_{k-1}, \delta]$, where $\delta=1$ if $\operatorname{det}(M)$ is a square in $\mathbb{F}_{q}$, and $\delta$ is any nonsquare in $\mathbb{F}_{q}$ if $\operatorname{det}(M)$

is a nonsquare in $\mathbb{F}_{q}$.

The following theorem presents a characterization of LCD codes in terms of their basis.

Theorem 5.2: Let $q$ be a power of an odd prime and $\mathcal{C}$ be an $[n, k]$ LCD code over $\mathbb{F}_{q}$. Then, $\mathcal{C}$ is LCD if and only if there is a generator matrix $G$ of $\mathcal{C}$ such that $G G^{T}=\operatorname{diag}[\underbrace{1,1, \ldots, 1}_{k-1}, \delta]$, where $\delta \in \mathbb{F}_{q}^{*}=\mathbb{F}_{q} \backslash\{0\}$, that is, there is a basis $\mathbf{e}_{1}, \ldots, \mathbf{e}_{k}$ of $\mathcal{C}$ such that for any $i, j \in\{1,2, \ldots, k\}$,

(i) $\mathbf{e}_{i} \cdot \mathbf{e}_{j}=0$ if $i \neq j$;

(ii) $\mathbf{e}_{i} \cdot \mathbf{e}_{i}=1$ if $i \neq k$;

(ii) $\mathbf{e}_{k} \cdot \mathbf{e}_{k}=\delta$.

Proof Using Proposition 5.1, we can prove this theorem by a similar discussion as in the proof of Theorem 3.1 and Theorem 3.2

Let $G_{1}$ and $G_{2}$ be any two generator matrices of an $[n, k]$ code $\mathcal{C}$. Then, there is a $k \times k$ nonsingular matrix $Q$ such that $G_{2}=$ $Q G_{1}$. Thus, $\operatorname{det}\left(G_{2} G_{2}^{T}\right)=\operatorname{det}(Q)^{2} \operatorname{det}\left(G_{1} G_{1}^{T}\right)$. Then, $\operatorname{det}\left(G_{2} G_{2}^{T}\right) \operatorname{det}\left(G_{1} G_{1}^{T}\right)^{-1} \in\left(\mathbb{F}_{q}^{*}\right)^{2}$. Hence, we can define LCD $+[n, k]_{q}$ $\left(\mathrm{LCD}_{-}[n, k]_{q}\right.$, respectively) be the set of all $[n, k]$ LCD codes over $\mathbb{F}_{q}$ with $\operatorname{det}\left(G G^{T}\right) \in\left(\mathbb{F}_{q}^{*}\right)^{2}\left(\operatorname{det}\left(G G^{T}\right) \notin\left(\mathbb{F}_{q}^{*}\right)^{2}\right.$, respectively). Let $\operatorname{LCD}[n, k]_{q}$ be the set of all $[n, k] \operatorname{LCD}$ codes over $\mathbb{F}_{q}$. Then, $\operatorname{LCD}[n, k]_{q}=\mathrm{LCD}_{+}[n, k]_{q} \cup \mathrm{LCD}_{-}[n, k]_{q}$. To construct linear codes in $\mathrm{LCD}_{-}[n, k]_{q}$, one need the following lemma [15].

Lemma 5.3: For any $z \in \mathbb{F}_{q}$, there exist $x$ and $y$ in $\mathbb{F}_{q}$ such that $z=x^{2}+y^{2}$.

Let $\gamma$ be a nonsquare in $\mathbb{F}_{q}$. From Lemma 5.3, there exist $a$ and $b$ in $\mathbb{F}_{q}$ such that $\gamma=a^{2}+b^{2}$. Let $G_{+}$and $G_{-}$be $k \times n$ matrices defined by

$$
G_{+}=\left[\begin{array}{c}
\mathbf{e}_{1} \\
\mathbf{e}_{2} \\
\vdots \\
\mathbf{e}_{k-1} \\
\mathbf{e}_{k}
\end{array}\right] \text { and } G_{-}=\left[\begin{array}{c}
\mathbf{e}_{1} \\
\mathbf{e}_{2} \\
\vdots \\
\mathbf{e}_{k-1} \\
a \mathbf{e}_{k}+b \mathbf{e}_{k+1}
\end{array}\right]
$$

Let $\mathcal{C}_{+}$and $\mathcal{C}_{-}$be linear codes generated by $G_{+}$and $G_{-}$respectively. Since $G_{+} G_{+}^{T}=I_{k}$ and $G_{-} G_{-}^{T}=\operatorname{diag}[\underbrace{1,1, \ldots, 1}_{k-1}, \gamma]$, $\mathcal{C}_{+} \in \mathrm{LCD}_{+}[n, k]_{q}$ and $\mathcal{C}_{-} \in \mathrm{LCD}_{-}[n, k]_{q}$. Moreover, $\mathcal{C}_{+}$and $\mathcal{C}_{-}$have parity-check matrices $H_{+}$and $H_{-}$respectively, where

$$
H_{+}=\left[\begin{array}{c}
\mathbf{e}_{k+1} \\
\mathbf{e}_{k+2} \\
\vdots \\
\mathbf{e}_{n-1} \\
\mathbf{e}_{n}
\end{array}\right] \text { and } H_{-}=\left[\begin{array}{c}
\mathbf{e}_{k+2} \\
\mathbf{e}_{k+3} \\
\vdots \\
\mathbf{e}_{n} \\
-b \mathbf{e}_{k}+a \mathbf{e}_{k+1}
\end{array}\right]
$$

Then, $\mathcal{C}_{+}^{\perp} \in \mathrm{LCD}_{+}[n, n-k]_{q}$ and $\mathcal{C}_{-}^{\perp} \in \mathrm{LCD}_{-}[n, n-k]_{q}$. Further, we have the following results.

Proposition 5.4: Let $0<k<n$. If $\mathcal{C} \in \mathrm{LCD}_{+}[n, n-k]_{q}\left(\mathcal{C} \in \mathrm{LCD}_{-}[n, n-k]_{q}\right.$, respectively), then $\mathcal{C}^{\perp} \in \mathrm{LCD}_{+}[n, n-k]_{q}$ $\left(\mathcal{C}^{\perp} \in \mathrm{LCD}_{-}[n, n-k]_{q}\right.$, respectively).

Proof Let $G$ be a generator matrix of $\mathcal{C}$ and $H$ be a parity-check matrix of $\mathcal{C}$. Note that $G H^{T}=0$. Then,

$$
\begin{aligned}
\left(\operatorname{det}\left(\left[\begin{array}{l}
G \\
H
\end{array}\right]\right)\right)^{2} & =\operatorname{det}\left(\left[\begin{array}{c}
G \\
H
\end{array}\right]\left[\begin{array}{c}
G \\
H
\end{array}\right]^{T}\right) \\
& =\operatorname{det}\left(\left[\begin{array}{cc}
G G^{T} & 0 \\
0 & H H^{T}
\end{array}\right]\right) \\
& =\operatorname{det}\left(G G^{T}\right) \operatorname{det}\left(H H^{T}\right) .
\end{aligned}
$$

Then, $\operatorname{det}\left(G G^{T}\right) \operatorname{det}\left(H H^{T}\right) \in\left(\mathbb{F}_{q}^{*}\right)^{2}$, which completes the proof.

Let $\mathbb{O}_{n}(q)$ be the set of all $n \times n$ matrix $Q$ over $\mathbb{F}_{q}$ such that $Q Q^{T}=I_{n}$. It is observed that $\mathbb{O}_{n}(q)$ acts on LCD $[n, n-k]_{q}$ by $(\mathcal{C}, Q) \longmapsto \mathcal{C} Q$, where $\mathcal{C} \in \operatorname{LCD}[n, k]_{q}$ and $Q \in \mathbb{O}_{n}(q)$. Moreover, LCD $+[n, k]_{q}$ and LCD $-[n, k]_{q}$ are $\mathbb{O}_{n}(q)$-invariant subsets. In fact, we have the following stronger results. 
Proposition 5.5: Let $k$ and $n$ be two positive integers with $k<n$. Then, $\operatorname{LCD}_{+}[n, k]_{q}=\mathcal{C}_{+} \mathbb{O}_{n}(q)$ and LCD_ $[n, k]_{q}=$ $\mathcal{C}_{-} \mathbb{O}_{n}(q)$. Hence, $\operatorname{LCD}[n, k]_{q}=\mathcal{C}_{+} \mathbb{O}_{n}(q) \cup \mathcal{C}_{-} \mathbb{O}_{n}(q)$.

Proof From $\mathrm{LCD}[n, k]_{q}=\mathrm{LCD}_{+}[n, k]_{q} \cup \mathrm{LCD}_{-}[n, k]_{q}$ and Proposition 5.4, this proposition follows.

Lemma 5.6: Let $\mathcal{C}$ be an $[n, k]$ LCD code over $\mathbb{F}_{q}, G$ be a generator matrix of $\mathcal{C}$ and $H$ be a generator matrix of $\mathcal{C}^{\perp}$. Then, $Q \in \operatorname{St}(\mathcal{C})$ if and only if $Q=\left[\begin{array}{c}G \\ H\end{array}\right]^{-1}\left[\begin{array}{cc}Q_{1} & 0 \\ 0 & Q_{2}\end{array}\right]\left[\begin{array}{c}G \\ H\end{array}\right]$, where $Q_{1} \in \mathbb{G L}_{k}(q)$ and $Q_{2} \in \mathbb{G L}_{n-k}(q)$ such that $Q_{1}\left(G G^{T}\right) Q_{1}^{T}=G G^{T}$ and $Q_{2}\left(H H^{T}\right) Q_{2}^{T}=H H^{T}$.

Proof The proof is analogous to the proof of Lemma 4.4

For $\delta \in \mathbb{F}_{q}^{*}$, let $\mathbb{O}_{k}^{\delta}(q)$ be the group defined by

$$
\mathbb{O}_{k}^{\delta}(q)=\left\{Q \in \mathbb{O}_{k}(q): Q \operatorname{diag}[\underbrace{1,1, \ldots, 1}_{k-1}, \delta] Q^{T}=\operatorname{diag}[\underbrace{1,1, \ldots, 1}_{k-1}, \delta]\right\} .
$$

Then, $\mathbb{O}_{k}^{1}(q)$ is just $\mathbb{O}_{k}(q)$.

Corollary 5.7: Let $k$ and $n$ be two positive integers with $0<k<n$.

(i) Let $\mathcal{C}_{+}$be the LCD code with the generator matrix $G_{+}$defined by Equation (10). Then,

$$
\operatorname{St}\left(\mathcal{C}_{+}\right)=\left\{\left[\begin{array}{cc}
Q_{1} & 0 \\
0 & Q_{2}
\end{array}\right]: Q_{1} \in \mathbb{O}_{k}(q), Q_{2} \in \mathbb{O}_{n-k}(q)\right\} .
$$

(ii) Let $G_{-}$and $H_{-}$be matrices defined by Equations (10) and (11). Then,

$$
\operatorname{St}\left(\mathcal{C}_{-}\right)=\left\{\left[\begin{array}{l}
G_{-} \\
H_{-}
\end{array}\right]^{-1}\left[\begin{array}{cc}
Q_{1} & 0 \\
0 & Q_{2}
\end{array}\right]\left[\begin{array}{c}
G_{-} \\
H_{-}
\end{array}\right]: Q_{1} \in \mathbb{O}_{k}^{\gamma}(q), Q_{2} \in \mathbb{O}_{n-k}^{\gamma}(q)\right\} .
$$

Proof This corollary follows from Lemma 5.6

To determine the cardinality of the orbit $\mathcal{C} \mathbb{O}_{n}(q)$, we need the cardinality of $\mathbb{O}_{n}^{\delta}(q)$, which can be found in [13], [16]. If $n$ is odd, one has

$$
\left|\mathbb{O}_{n}^{\delta}(q)\right|=2 q^{\frac{(n-1)^{2}}{4}} \prod_{i=1}^{\frac{n-1}{2}}\left(q^{2 i}-1\right)
$$

If $n$ is even, one has

$$
\left|\mathbb{O}_{n}^{\delta}(q)\right|=2 q^{\frac{n(n-2)}{4}}\left(q^{\frac{n}{2}}-\eta\left((-1)^{\frac{n}{2}} \delta\right)\right) \prod_{i=1}^{\frac{n}{2}-1}\left(q^{2 i}-1\right),
$$

where $\eta$ is the Legendre character of $\mathbb{F}_{q}$.

Theorem 5.8: Let $q$ be a power of odd prime and $k, n$ be two positive integers with $k<n$.

(i) $\mathrm{LCD}_{+}[n, k]_{q}=\mathcal{C}_{+} \mathbb{O}_{n}(q)$ and

$$
\left|\mathrm{LCD}_{+}[n, k]_{q}\right|= \begin{cases}\frac{1}{2} q^{\frac{k(n-k)-1}{2}}\left(q^{\frac{n}{2}}-\eta\left((-1)^{\frac{n}{2}}\right)\right)\left[\begin{array}{l}
\frac{n}{2}-1 \\
\frac{k-1}{2}
\end{array}\right]_{q^{2}}, & \text { if } k \text { odd, } n \text { even, } \\
\frac{1}{2} q^{\frac{k(n-k)}{2}}\left(q^{\frac{n-k}{2}}+\eta\left((-1)^{\frac{n-k}{2}}\right)\right)^{\left[\frac{n-1}{2}\right.}\left[\frac{k-1}{2}\right]_{q^{2}}, & \text { if } k \text { odd, } n \text { odd, } \\
\frac{1}{2} q^{\frac{k(n-k)}{2}}\left(q^{\frac{k}{2}}+\eta\left((-1)^{\frac{k}{2}}\right)\right)\left[\begin{array}{l}
\frac{n-1}{2} \\
\frac{k}{2}
\end{array} q^{2},\right. & \text { if } k \text { even, } n \text { odd, } \\
\frac{1}{2} q^{\frac{k(n-k)}{2}} \frac{\left(q^{\frac{k}{2}}+\eta\left((-1)^{\frac{k}{2}}\right)\right)\left(q^{\frac{n-k}{2}}+\eta\left((-1)^{\frac{n-k}{2}}\right)\right)}{\left(q^{\frac{n}{2}}+\eta\left((-1)^{\frac{n}{2}}\right)\right)}\left[\begin{array}{l}
\frac{n}{2} \\
\frac{k}{2}
\end{array} q^{2},\right. & \text { if } k \text { even, } n \text { even. }\end{cases}
$$

(ii) $\mathrm{LCD}_{-}[n, k]_{q}=\mathcal{C}_{-} \mathbb{O}_{n}(q)$ and

$$
\left|\mathrm{LCD}_{-}[n, k]_{q}\right|= \begin{cases}\frac{1}{2} q^{\frac{k(n-k)-1}{2}}\left(q^{\frac{n}{2}}-\eta\left((-1)^{\frac{n}{2}}\right)\right)^{\left[\begin{array}{l}
\frac{n}{2}-1 \\
\frac{k-1}{2}
\end{array}\right]_{q^{2}},} & \text { if } k \text { odd, } n \text { even, } \\
\left.\frac{1}{2} q^{\frac{k(n-k)}{2}}\left(q^{\frac{n-k}{2}}-\eta\left((-1)^{\frac{n-k}{2}}\right)\right)^{\left[\frac{n-1}{2}\right.}\right]_{\frac{k-1}{2}}, & \text { if } k \text { odd, } n \text { odd, } \\
\frac{1}{2} q^{\frac{k(n-k)}{2}}\left(q^{\frac{k}{2}}-\eta\left((-1)^{\frac{k}{2}}\right)\right)\left[\begin{array}{l}
\frac{n-1}{2} \\
\frac{k}{2}
\end{array}\right]_{q^{2}}, & \text { if } k \text { even, } n \text { odd, } \\
\frac{1}{2} q^{\frac{k(n-k)}{2}} \frac{\left(q^{\frac{k}{2}}-\eta\left((-1)^{\frac{k}{2}}\right)\right)\left(q^{\frac{n-k}{2}}-\eta\left((-1)^{\frac{n-k}{2}}\right)\right)}{\left(q^{\frac{n}{2}}+\eta\left((-1)^{\frac{n}{2}}\right)\right)}\left[\begin{array}{l}
\frac{n}{2} \\
\frac{k}{2}
\end{array} q^{2},\right. & \text { if } k \text { even, } n \text { even. }\end{cases}
$$


Proof From $\mathrm{LCD}_{+}[n, k]_{q}=\mathcal{C}_{+} \mathbb{O}_{n}(q)$ and Corollary 5.7, one has

$$
\begin{aligned}
\left|\mathrm{LCD}_{+}[n, k]_{q}\right| & =\frac{\left|\mathbb{O}_{n}(q)\right|}{\left|\operatorname{St}\left(\mathcal{C}_{+}\right)\right|} \\
& =\frac{\left|\mathbb{O}_{n}(q)\right|}{\left|\mathbb{O}_{k}(q)\right| \cdot\left|\mathbb{O}_{n-k}(q)\right|} .
\end{aligned}
$$

Then, Part (i) follows from Equations (12) and (13).

From LCD ${ }_{-}[n, k]_{q}=\mathcal{C}_{-} \mathbb{O}_{n}(q)$ and Corollary [5.7, one has

$$
\begin{aligned}
\left|\operatorname{LCD}_{-}[n, k]_{q}\right| & =\frac{\left|\mathbb{O}_{n}(q)\right|}{\left|\operatorname{St}\left(\mathcal{C}_{-}\right)\right|} \\
& =\frac{\left|\mathbb{O}_{n}(q)\right|}{\left|\mathbb{O}_{k}^{\gamma}(q)\right| \cdot\left|\mathbb{O}_{n-k}^{\gamma}(q)\right|} .
\end{aligned}
$$

Then, Part (ii) follows from Equations (12) and (13).

Corollary 5.9: Let $q$ be a power of an odd prime and $k, n$ be two positive integers with $k<n$. Then

$$
\left|\operatorname{LCD}[n, k]_{q}\right|= \begin{cases}q^{\frac{k(n-k)-1}{2}}\left(q^{\frac{n}{2}}-\eta\left((-1)^{\frac{n}{2}}\right)\right)\left[\begin{array}{l}
\frac{n}{2}-1 \\
\frac{k-1}{2}
\end{array}\right]_{q^{2}}, & \text { if } k \text { odd, } n \text { even, } \\
\left.q^{\frac{(k+1)(n-k)}{2}\left[\frac{n-1}{2}\right.}\right]_{q^{2}}, & \text { if } k \text { odd, } n \text { odd, } \\
\left.q^{\frac{k(n-k+1)}{2}\left[\frac{n-1}{2}\right.}\right]_{q^{2}}, & \text { if } k \text { even, } n \text { odd, } \\
q^{\frac{k(n-k)}{2}\left[\frac{n}{2}\right.}\left[\frac{k}{2}\right]_{q^{2}}, & \text { if } k \text { even, } n \text { even. }\end{cases}
$$

Proof This corollary follows from Theorem 5.8

Corollary 5.10: Let $k$ and $n$ be positive integers with $k<n$.

(i) $\mathrm{LCD}_{+}[n, k]_{q}=\mathcal{C}_{+} \mathbb{O}_{n}(q)$ and

$$
\lim _{\substack{k \rightarrow \infty \\
(n-k) \rightarrow \infty}} \frac{\left|\mathrm{LCD}_{+}[n, k]_{q}\right|}{\left[\begin{array}{l}
n \\
k
\end{array}\right]_{q}}=\frac{1}{2 \prod_{i=1}^{\infty}\left(1+\frac{1}{q^{i}}\right)} .
$$

(ii) $\mathrm{LCD}_{-}[n, k]_{q}=\mathcal{C}_{-} \mathbb{O}_{n}(q)$ and

$$
\lim _{\substack{k \rightarrow \infty \\
(n-k) \rightarrow \infty}} \frac{\left|\mathrm{LCD}_{-}[n, k]_{q}\right|}{\left[\begin{array}{l}
n \\
k
\end{array}\right]_{q}}=\frac{1}{2 \prod_{i=1}^{\infty}\left(1+\frac{1}{q^{i}}\right)} .
$$

(iii) Let $\operatorname{LCD}[n, k]_{q}$ be the set of all $[n, k]$ LCD codes over $\mathbb{F}_{q}$. Then,

$$
\lim _{\substack{k \rightarrow \infty \\
(n-k) \rightarrow \infty}} \frac{\left|\mathrm{LCD}[n, k]_{q}\right|}{\left[\begin{array}{l}
n \\
k
\end{array}\right]_{q}}=\frac{1}{\prod_{i=1}^{\infty}\left(1+\frac{1}{q^{i}}\right)} .
$$

Proof From Theorem 5.8 and Equation (8), it completes the proof.

Corollary 5.11: Let $k$ and $n$ be two positive integers with $k<n$. Then,

$$
\lim _{\substack{k \rightarrow \infty \\(n-k) \rightarrow \infty}} \frac{\left|\mathrm{LCD}_{+}[n, k]_{q}\right|}{\left|\mathrm{LCD}[n, k]_{q}\right|}=\lim _{\substack{k \rightarrow \infty \\(n-k) \rightarrow \infty}} \frac{\left|\mathrm{LCD}_{-}[n, k]_{q}\right|}{\left|\mathrm{LCD}[n, k]_{q}\right|}=\frac{1}{2} .
$$

Thus,

$$
\lim _{\substack{k \rightarrow \infty \\(n-k) \rightarrow \infty}} \frac{\left|\mathrm{LCD}_{+}[n, k]_{q}\right|}{\left|\mathrm{LCD}_{-}[n, k]_{q}\right|}=1 .
$$

Proof This corollary follows from Corollary 5.10

By a similar discussion as the binary case, classifying the inequivalent $q$-array LCD codes is equivalent to determining representatives of the following two sets of double cosets:

$$
\operatorname{St}\left(\mathcal{C}_{+}\right) \backslash \mathbb{O}_{n}(q) / \mathbb{P}_{n} \text { and } \operatorname{St}\left(\mathcal{C}_{-}\right) \backslash \mathbb{O}_{n}(q) / \mathbb{P}_{n}
$$




\section{CONCLUDING REMARKS}

In this paper, we have pushed further the study of the general structure of LCD codes. Firstly, we have provided a new characterization of LCD codes by their basis. As a consequence, a conjecture on minimum distance of binary LCD codes proposed by Galvez et al. [7] was solved. Then, we have considered the action of the orthogonal group on the set of all LCD codes. All the possible orbits of this action have been identified and closed formulas of the size of the orbits have been derived. Our results show that almost all binary LCD codes are odd-like codes with odd-like duals and about half of $q$-LCD codes have orthonormal basis, where $q$ is a power of an odd prime.

\section{REFERENCES}

[1] J. Bringer, C. Carlet, H. Chabanne, S. Guilley, and H. Maghrebi. Orthogonal direct sum masking a smartcard friendly computation paradigm in a code, with builtin protection against side-channel and fault attacks. In WISTP, volume 8501 of Lecture Notes in Comput. Sci., pages $40-56$. Springer, Berlin, 2014.

[2] C. Carlet and S. Guilley. Complementary dual codes for counter- measures to side-channel attacks. In Proceedings of the 4th ICMCTA Meeting, volume 3 of CIM Series in Mathematical Sciences book series, pages 87-95. Springer, Berlin, 2014.

[3] C. Carlet, S. Mesnager, C. Tang and Y. Qi, Linear codes over $\mathbb{F}_{q}$ which are equivalent to LCD codes, arXiv preprint arXiv:1703.04346 2017.

[4] C. Carlet, S. Mesnager, C. Tang and Y. Qi, On $\sigma$-LCD codes , arXiv preprint arXiv:1707.08789. 2017.

[5] C. Li, C. Ding and S. Li. LCD Cyclic codes over finite fields. IEEE Trans. Inf. Theory, vol. 63, no. 7, pp. 4344 - $4356,2017$.

[6] S. T. Dougherty, J. L. Kim, B. Ozkaya, L. Sok and P. Solé. The combinatorics of LCD codes: Linear Programming bound and orthogonal matrices. International Journal of Information and Coding Theory, 4(2-3), 116-128, 2017.

[7] L. Galvez, J. L. Kim, N. Lee, Y. G. Roe, and B. S. Won. Some Bounds on Binary LCD Codes. arXiv preprint arXiv:1701.04165 2017.

[8] J. F. Humphreys. A course in group theory. Oxford Science Publications, The Clarendon Press, Oxford University Press, New York, 1996.

[9] S. Li, C. Li, C. Ding, and H. Liu, Two Families of LCD BCH Codes. IEEE Trans. Inf. Theory, vol 63, no. 9, pp. 5699 - 5717, 2017.

[10] S. Mesnager, C. Tang and Y. Qi, Complementary dual algebraic geometry codes, arXiv preprint arXiv:1609.05649 2016.

[11] James L. Massey. Linear codes with complementary duals. Discrete Mathematics, 106-107:337-342, 1992.

[12] J. MacWilliams. Orthogonal matrices over finite fields. Am. Math. Monthly 76, 152-164 (1969).

[13] Sheekey, J.; On rank problems for subspaces of matrices over finite fields, Ph.D. thesis.

[14] N. Sendrier. On the dimension of the hull. SIAM Journal on Discrete Mathematics, 10(2), 282-293, 1997.

[15] J. P. Serre. A course in arithmetic. Springer Science \& Business Media, 2012.

[16] H. Weyl. The classical groups: their invariants and representations. Princeton university press, 2016. 